\title{
NONCONFORMING MULTISCALE FINITE ELEMENT METHOD FOR STOKES FLOWS IN HETEROGENEOUS MEDIA. PART I: METHODOLOGIES AND NUMERICAL EXPERIMENTS*
}

\author{
B. P. MULJADI ${ }^{\dagger}$, J. NARSKI ${ }^{\ddagger}$, A. LOZINSKI ${ }^{\S}$, AND P. DEGOND $\ddagger$
}

\begin{abstract}
The multiscale finite element method (MsFEM) is developed in the vein of the Crouzeix-Raviart element for solving viscous incompressible flows in genuine heterogeneous media. Such flows are relevant in many branches of engineering, often at multiple scales and at regions where analytical representations of the microscopic features of the flows are often unavailable. Full accounts of these problems heavily depend on the geometry of the system under consideration and are computationally expensive. Therefore, a method capable of solving multiscale features of the flow without confining itself to fine scale calculations is sought. The approximation of boundary condition on coarse element edges when computing the multiscale basis functions critically influences the eventual accuracy of any MsFEM approaches. The weakly enforced continuity of Crouzeix-Raviart function space across element edges leads to a natural boundary condition for the multiscale basis functions which relaxes the sensitivity of our method to complex patterns of obstacles exempt from the need to implement any oversampling techniques. Additionally, the application of a penalization method makes it possible to avoid a complex unstructured domain and allows extensive use of simpler Cartesian meshes.
\end{abstract}

Key words. Crouzeix-Raviart element, multiscale finite element method, Stokes equations, penalization method

AMS subject classifications. 35J15, 65N12, 65N30

DOI. $10.1137 / 14096428 \mathrm{X}$

1. Introduction. Stokes equations relate to many engineering practices such as reservoir engineering, micro-/nano-fluidics, and mechano-biological systems. Often in these fields, the problems are at multiple scales both spatially and temporally. Multiscale problems may arise due to highly oscillatory coefficients of the system or due to heterogeneity of the domain, for example, complex rock matrices when modelling subsurface flows or random placements of buildings, people, and trees in the context of urban canopy flows. It is very difficult to resolve numerically all of the scales that impact transport through such systems. Despite the modern renaissance of high performance computing, the size of the discrete problems remains intractable. In some engineering contexts, it is sometimes sufficient to predict macroscopic properties of multiscale systems. Hence, it is desirable to develop an efficient computational algorithm to solve multiscale problems without being confined to solving fine scale solutions. We borrow the concept of the multiscale finite element method (MsFEM)

*Received by the editors April 9, 2015; accepted for publication (in revised form) August 5, 2015; published electronically October 22, 2015. This work was done under the auspices of "Fondation Sciences et Technologies pour l'Aeronautique et l'Espace," in the frame of the project 'AGREMEL' (contract RTRA-STAE/2011/AGREMEL/02).

http://www.siam.org/journals/mms/13-4/96428.html

†Université de Toulouse, UPS, INSA, UT1, UTM, Institut de Mathématiques de Toulouse, F31062 Toulouse, France, and CNRS, Institut de Mathématiques de Toulouse UMR 5219, F-31062 Toulouse, France. Current address: Department of Earth Science and Engineering, Imperial College London, London SW7 2BP, UK (muljadi.bp@gmail.com).

$\ddagger$ Université de Toulouse, UPS, INSA, UT1, UTM, Institut de Mathématiques de Toulouse, F31062 Toulouse, France, and CNRS, Institut de Mathématiques de Toulouse UMR 5219, F-31062 Toulouse, France (jacek.narski@gmail.com, pdegond@imperial.ac.uk).

$\S$ Laboratoire de Mathématiques de Besançon, UMR CNRS 6623, Université de Franche-Comté, 25030 Besançon Cedex, France (alozinski@gmail.com). 
[1] by Hou and $\mathrm{Wu}$, the concept of which hinges upon the extension of multiscale basis functions precomputed in fine mesh to represent a "model" of the microscopic structure of the flow. The fact that the multiscale bases are not modeled but rather computed extends the applicability of MsFEM to problems where the analytical representations of microscopic solutions are unavailable.

Within the past decades, several similar methods have appeared, namely, generalized finite element methods [2], the wavelet-based homogenization method [3], the variational multiscale method [4], various methods derived from homogenization theory [5], equation-free computations [6], the heterogeneous multiscale method [7], and many others. In the context of diffusion in perforated media, some studies have been done both theoretically and numerically in [8], [9], [10], [11], and [12]. For the case of advection-diffusion, a method derived from the heterogeneous multiscale method addressing oscillatory coefficients is studied, for example, in [13], [14], [15]. For viscous, incompressible flows, multiscale methods based on homogenization theory for solving slowly varying Stokes flow in porous media have been studied in [16], [17]. Several theoretical and numerical studies have been done in recent years to address Stokes-Darcy or Stokes-Brinkman problems in vugular or fractured porous media; see [18], [19], [20], [21].

When tackling highly heterogeneous problems, it is understood that a delicate treatment is needed at the boundary condition when constructing the multiscale basis function because it greatly influences the eventual accuracy of the method under consideration. Indeed, in the original work of $\mathrm{Hou}$ and $\mathrm{Wu}$, the oversampling method was introduced to provide the best approximation of the boundary condition of the multiscale basis functions. Inaccurate approximations of these functions on the boundary would leave a method highly unreliable when dealing with arbitrary pattern of porosities or matrices. Oversampling here means that the local problems in the coarse element are extended to a domain larger than the element itself, but only the interior information would be communicated to the coarse scale equation. The aim is to reduce the effect of wrong boundary conditions and bad sampling sizes. The ways in which the sampled domain is extended lead to various oversampling methods; we refer interested readers to [22], [23], [24], [25].

The nonconforming nature of the Crouzeix-Raviart element (see [26]) is shown to provide great "flexibility" especially when arbitrary patterns of porosities are considered. In the construction of Crouzeix-Raviart multiscale basis functions, the conformity between coarse elements is not enforced in a strong sense but rather in a weak sense; i.e., the method requires merely the average of the "jump" of the function to vanish at coarse element edges. When very dense obstacles are introduced, which often makes it prohibitively difficult to avoid intersections between coarse element edges and obstacles, the benefit of using the Crouzeix-Raviart MsFEM is significant because it allows the multiscale basis functions to have natural boundary conditions on element edges, making it insensitive to complex patterns of obstacles. Moreover, the integrated application of a penalization method enables one to carry the simulations onto simple Cartesian meshes. This work continues our earlier works, where the Crouzeix-Raviart MsFEM is implemented on diffusion and advection-diffusion problems [27], [28], [29].

This paper is organized as follows. The formulation of the problem is given in section 2. The heart of our paper is laid out in section 3, where the Crouzeix-Raviart MsFEM is explained. In section 4, the adaptation of a penalization method to our problem is discussed. Numerical tests comprising enclosed and open-channel flows 


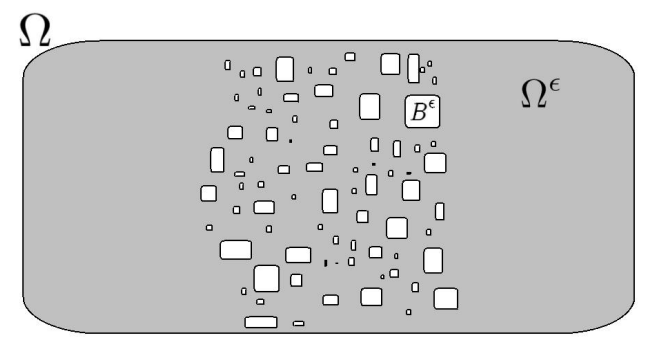

FIG. 1. An illustration of the domain $\Omega$ comprising the fluid domain $\Omega^{\epsilon}$ perforated by a set of obstacles $B^{\epsilon}$.

with an arbitrary pattern of obstacles and nonhomogeneous boundary conditions are reported in section 5 , followed by some concluding remarks.

2. Formulation of the problem. We consider a Stokes problem posed in a bounded domain $\Omega \subset \mathbb{R}^{d}$ within which a set $B^{\epsilon}$ of obstacles is included. The domain with voids left by obstacles is denoted by $\Omega^{\epsilon}=\Omega \backslash B^{\epsilon}$ illustrated in Figure 1, where $\epsilon$ denotes the minimum width of obstacles. The Stokes problem is then to find $u$ : $\Omega^{\epsilon} \rightarrow \mathbb{R}$, which is the solution to

$$
\begin{aligned}
-\nu \Delta \vec{u}+\nabla p=\vec{f} \quad \text { in } \quad \Omega^{\epsilon}, \\
\nabla \cdot \vec{u}=0 \quad \text { in } \quad \Omega^{\epsilon} .
\end{aligned}
$$

The boundary value problem considered in (2.1) posed on two-dimensional domain $\Omega$, together with boundary condition on $\partial \Omega$, is given by

$$
\begin{array}{lll}
\vec{u}=0 & \text { on } & \partial B^{\epsilon} \cap \partial \Omega^{\epsilon}, \\
\vec{u}=\vec{w} & \text { on } & \partial \Omega \cap \partial \Omega^{\epsilon},
\end{array}
$$

where $\vec{f}: \Omega \rightarrow \mathbb{R}$ is a given function, and $\vec{w}$ is a function fixed on boundary $\partial \Omega$. In this paper, we consider only the Dirichlet boundary condition on $\partial B^{\epsilon}$, namely $u_{\mid \partial B^{\epsilon}}=$ 0 , thereby assuming that the obstacle is impenetrable. Other kinds of boundary conditions on $\partial B^{\epsilon}$ are subject to a completely new endeavor.

The weak formulation. Let us restrict ourselves to the case of homogeneous boundary conditions $\vec{w}=0 .{ }^{1}$ Introduce the function spaces $V=\left(H_{0}^{1}\left(\Omega^{\epsilon}\right)\right)^{d}$ for the velocity, $M=L_{0}^{2}\left(\Omega^{\varepsilon}\right)=\left\{p \in L^{2}\left(\Omega^{\varepsilon}\right)\right.$ s.t. $\left.\int_{\Omega^{\varepsilon}} p=0\right\}$ for the pressure, and $X=V \times M$. The weak formulation of the problem above reads as follows: find $(\vec{u}, p) \in X$ such that

$$
c((\vec{u}, p),(\vec{v}, q))=\int_{\Omega^{\varepsilon}} \vec{f} \cdot \vec{v} \quad \forall(\vec{v}, q) \in X
$$

where $c$ is the bilinear form defined by

$$
c((\vec{u}, p),(\vec{v}, q))=\int_{\Omega^{\varepsilon}} \nabla \vec{u}: \nabla \vec{v}-\int_{\Omega^{\varepsilon}} p \operatorname{div} \vec{v}-\int_{\Omega^{\varepsilon}} q \operatorname{div} \vec{u} .
$$

\footnotetext{
${ }^{1}$ This is done only to simplify the forthcoming presentation of our MsFEM technique. Its generalization to nonhomogeneous boundary data is straightforward and is explained in detail in section 4.3 .
} 
The existence and uniqueness of the solution to problem (2.3) is guaranteed by the theory of saddle point problems, especially by the inf-sup property

$$
\inf _{p \in M} \sup _{\vec{v} \in V} \frac{\int_{\Omega^{\varepsilon}} p \operatorname{div} \vec{v}}{\|p\|_{M}\|\vec{v}\|_{V}} \geq \gamma>0
$$

which is known to hold on any domain with Lipschitz boundary [30]. A nice reformulation of this theory is presented in [31], where it is proved that (2.4) implies also the inf-sup property for the form $c$,

$$
\inf _{(\vec{u}, p) \in X} \sup _{(\vec{v}, q) \in X} \frac{c((\vec{u}, p),(\vec{v}, q))}{\|\vec{u}, p\|_{X}\|\vec{v}, q\|_{X}} \geq \gamma_{c}>0,
$$

with a constant $\gamma_{c}$ that depends only on the constant $\gamma$ in (2.4). One then invokes the BNB theorem, which states that the variational problem (2.3) has the unique solution provided the symmetric bilinear form $c$ is bounded (which is evident in our case) and satisfies the inf-sup property (2.5). Note that the second hypothesis in the BNB theorem of [31] is void in the case of problems with a symmetric bilinear form.

Remark 2.1. In what follows, we shall sometimes extend the functions defined on $\Omega^{\epsilon}$ and vanishing on $\partial B^{\epsilon}$ to the whole domain $\Omega$ by setting them to 0 on $B^{\epsilon}$. From now on, we shall identify such functions with their extended versions without further notice. For example, an alternative definition of the space $V$ can be written as

$$
V=\left\{\vec{v} \in H_{0}^{1}(\Omega)^{d} \text { such that } \vec{v}=0 \text { on } B^{\epsilon}\right\} .
$$

3. Crouzeix-Raviart MsFEM space for Stokes equations. Let us introduce a mesh $\mathcal{T}_{H}$ on $\Omega$ consisting of $N_{H}$ polygons/polyhedrons of diameter at most $H$. Let $\mathcal{E}_{H}$ denote the set of all the edges/faces of $\mathcal{T}_{H}$ including those on the domain boundary $\partial \Omega$. It is assumed that the mesh does not contain any hanging nodes, and each edge is shared by two elements except those on $\partial \Omega$, which belong only to one element. We also assume that the mesh $\mathcal{T}_{H}$ is regular; i.e., for any mesh element $T \in \mathcal{T}_{H}$, there exists a smooth one-to-one mapping $\mathcal{M}: \tilde{T} \rightarrow T$, where $\tilde{T} \subset \mathbb{R}^{d}$ is the element of reference, and that $\|\nabla \mathcal{M}\|_{L^{\infty}} \leq D H,\left\|\nabla \mathcal{M}^{-1}\right\|_{L^{\infty}} \leq D H^{-1}$, with $D$ a universal constant independent of $T$. From now on, the elements of $\mathcal{T}_{H}$ will be referred to as the triangles, and the elements of $\mathcal{E}_{H}$ will be referred to as the edges, although all of the reasoning makes perfect sense in a general situation of any ambient dimension and a mesh consisting of polygons/polyhedrons of any shape.

3.1. Definition of the MsFEM space. To construct the MsFEM space, as done in the previous works of Le Bris, Legoll, and Lozinski [27], [28], we first introduce the extended velocity space

$$
V_{H}^{\text {ext }}=\left\{\begin{array}{c}
\vec{u} \in\left(L^{2}(\Omega)\right)^{d} \text { such that }\left.\vec{u}\right|_{T} \in\left(H^{1}(T)\right)^{d} \text { for any } T \in \mathcal{T}_{H}, \vec{u}=0 \text { on } B^{\epsilon}, \\
\int_{E}[[u]]=0 \forall E \in \mathcal{E}_{H},
\end{array}\right\}
$$

where $[[u]]$ denotes the jump of $u$ across an internal edge and $[[u]]=u$ on the boundary $\partial \Omega$. The idea behind this space is to enhance the natural velocity space $\left(H_{0}^{1}(\Omega)\right)^{d}$ so that we have at our disposal the vector fields discontinuous across the edges of the mesh, since our goal is to construct a nonconforming approximation method. The continuity is preserved only in the weak sense by requiring that the average be conserved across any edge. 
Now we want to decompose the extended velocity-pressure space $X_{H}^{e x t}=V_{H}^{e x t} \times M$ into the direct sum of a finite-dimensional subspace $X_{H}$ of coarse scales, which will be used for approximation, and an infinite-dimensional subspace $X_{H}^{0}$ of unresolved fine scales

$$
X_{H}^{e x t}=X_{H} \oplus X_{H}^{0}
$$

More specifically, we introduce the fine scale subspace as $X_{H}^{0}=V_{H}^{0} \times M_{H}^{0}$ with

$$
\begin{aligned}
V_{H}^{0} & =\left\{\vec{u} \in V_{H}^{\text {ext }} \text { such that } \int_{E} \vec{u}=0 \forall E \in \mathcal{E}_{H}\right\}, \\
M_{H}^{0} & =\left\{p \in M \text { such that } \int_{T} p=0 \forall T \in \mathcal{T}_{H}\right\} .
\end{aligned}
$$

The subspace $X_{H}$ is then defined as the "orthogonal" complement of $X_{H}^{0}$ with respect to the bilinear form $c$ :

$$
\left(\vec{u}_{H}, p_{H}\right) \in X_{H} \Longleftrightarrow c\left(\left(\vec{u}_{H}, p_{H}\right),(\vec{v}, q)\right)=0 \quad \forall(\vec{v}, q) \in X_{H}^{0} .
$$

Two remarks are in order to clarify this definition:

- The bilinear form $c$ is applied in the formula above to the functions from $V_{H}^{e x t}$, which are discontinuous across the edges of the mesh and thus nondifferentiable. To bypass this difficulty, the integrals in the definition of $c$ should be understood as $\int_{\Omega^{\varepsilon}} \cdots=\sum_{T \in \mathcal{T}_{H}} \int_{\Omega^{\varepsilon} \cap T} \cdots$. The same convention will be implicitly employed from now on if needed, as will be clear from the context.

- We have put the word "orthogonal" in quotes since the bilinear form $c$ is not a scalar product (not positive definite). However, we shall prove in the following lemma that the subspace $X_{H} \subset X_{H}^{e x t}$ defined by (3.2) indeed forms a direct sum with $X_{H}^{0}$.

Lemma 3.1. Let the functional spaces $M_{H} \subset M$ and $V_{H} \subset V_{H}^{\text {ext }}$ be defined as

$$
\begin{aligned}
& M_{H}=\left\{q \in L_{0}^{2}(\Omega) \text { such that }\left.q\right|_{T}=\text { const } \forall T \in \mathcal{T}_{H}\right\}, \\
V_{H}= & \left\{\vec{u}_{H}:\left(L^{2}(\Omega)\right)^{d}: \forall T \in \mathcal{T}_{H} \exists s \in L_{0}^{2}\left(\Omega^{\varepsilon} \cap T\right)\right. \text { such that } \\
& -\Delta \vec{u}_{H}+\nabla s=0 \text { on } \Omega^{\varepsilon} \cap T, \\
& \operatorname{div} \vec{u}_{H}=\text { const on } \Omega^{\varepsilon} \cap T, \\
& \vec{u}_{H}=0 \text { on } B^{\varepsilon} \cap T, \\
& \left.\nabla \vec{u}_{H} n-\text { sn }=\text { const on } E \cap \Omega^{\varepsilon} \forall E \in \mathcal{E}(T)\right\},
\end{aligned}
$$

where $\mathcal{E}(T)$ is the ensemble of edges composing $\partial T$. For any $\vec{u}_{H} \in V_{H}$, glue together the functions $s$ on triangles $T \in \mathcal{T}_{H}$ in the definition above into a single function $\pi_{H}\left(\vec{u}_{H}\right) \in M_{H}^{0}$ such that $\pi_{H}\left(\vec{u}_{H}\right)=s$ on any triangle $T \in \mathcal{T}_{H}$. Then $\pi_{H}: V_{H} \rightarrow M_{H}^{0}$ is a well defined linear operator, whose explicit formula is given in subsection 3.2. The space $X_{H}$ defined by (3.2) can be represented as

$$
X_{H}=\operatorname{span}\left\{\left(\vec{u}_{H}, \pi_{H}\left(\vec{u}_{H}\right)\right), \vec{u}_{H} \in V_{H}\right\} \oplus \operatorname{span}\left\{\left(0, \bar{p}_{H}\right), \bar{p}_{H} \in M_{H}\right\} .
$$

Moreover, the relation (3.1) holds true.

Proof. Let $\left(\vec{u}_{H}, p_{H}\right) \in X_{H}$ in the sense of definition (3.2). We can decompose $p_{H}$ as

$$
p_{H}=\bar{p}_{H}+p_{H}^{\prime} \text { with } \bar{p}_{H} \in M_{H} \text { and } p_{H}^{\prime} \in M_{H}^{0} \text {. }
$$

Copyright (c) by SIAM. Unauthorized reproduction of this article is prohibited. 
This decomposition is unique since the value $\bar{p}_{H}$ on any triangle $T \in \mathcal{T}_{H}$ is simply the average of $p_{H}$ on this triangle. Noting that

$$
\int_{\Omega^{\varepsilon}} \bar{p}_{H} \operatorname{div} \vec{v}=\left.\sum_{T \in \mathcal{T}_{H}} \bar{p}_{H}\right|_{T} \int_{T} \operatorname{div} \vec{v}=\left.\sum_{T \in \mathcal{T}_{H}} \bar{p}_{H}\right|_{T} \int_{\partial T} \bar{v} \cdot n=0
$$

for any $\bar{v} \in V_{H}^{0}$, we can rewrite definition (3.2) as

$$
c\left(\left(\vec{u}_{H}, p_{H}\right),(\vec{v}, q)\right)=\int_{\Omega^{\varepsilon}} \nabla \vec{u}_{H}: \nabla \vec{v}-\int_{\Omega^{\varepsilon}} p_{H}^{\prime} \operatorname{div} \vec{v}-\int_{\Omega^{\varepsilon}} q \operatorname{div} \vec{u}_{H}=0
$$

for any $\vec{v} \in V_{H}^{0}$ and $q \in M_{H}^{0}$.

Choosing a triangle $T \in \mathcal{T}_{H}$ and considering the test functions $\vec{v}=0, q \in L_{0}^{2}(T \cap$ $\left.\Omega^{\varepsilon}\right)$ with $q$ vanishing outside $T$ yields

$$
\int_{\Omega^{\varepsilon} \cap T} q \operatorname{div} \vec{u}_{H}=0 \quad \forall q \in L_{0}^{2}\left(T \cap \Omega^{\varepsilon}\right) \text {, i.e., } \operatorname{div} \vec{u}_{H}=\text { const on } T \cap \Omega^{\varepsilon} .
$$

We now observe that for any edge $E \in \mathcal{E}(T)$ there are functions $\vec{v}_{E, i}^{T} \in H^{1}(T)$, $i=1, \ldots, d$, such that $\vec{v}_{E, i}^{T}$ vanishes on $B^{\epsilon} \cap T$ and $\int_{E} \vec{v}_{E, i}=e_{i}$, with $\left\{e_{1}, \ldots, e_{d}\right\}$ being the canonical basis of $\mathbb{R}^{d}$, and $\int_{E^{\prime}} \vec{v}_{E, i}^{T}=0$ for all $E^{\prime} \in \mathcal{E}(T), E^{\prime} \neq E$. The space of functions in $H^{1}(T)$ vanishing on $B^{\epsilon} \cap T$ can be represented as

$$
\begin{aligned}
V(T) & :=\left\{\vec{v}:\left(H^{1}(T)\right)^{d} \text { such that } \vec{v}=0 \text { on } B^{\varepsilon} \cap T\right\} \\
& =V_{\int 0}(T) \oplus \operatorname{span}\left\{\vec{v}_{E, i}^{T}, E \in \mathcal{E}(T), i=1, \ldots, d\right\},
\end{aligned}
$$

where

$$
V_{\int 0}(T)=\left\{\vec{v}:\left(H^{1}(T)\right)^{d}: \int_{E} \vec{v}=0 \forall E \in \mathcal{E}(T) \text { and } \vec{v}=0 \text { on } B^{\varepsilon} \cap T\right\} .
$$

Denoting for any $E \in \mathcal{E}(T)$ and $i=1, \ldots, d$,

$$
\lambda_{E, i}^{T}=\int_{\Omega^{\varepsilon}} \nabla \vec{u}_{H}: \nabla \vec{v}_{E, i}^{T}-\int_{\Omega^{\varepsilon}} p_{H}^{\prime} \operatorname{div} \vec{v}_{E, i}^{T},
$$

and taking into account relation (3.6) with any $\vec{v} \in V_{\int 0}(T)$ extended by 0 outside $T$ and $q=0$, we see that

$$
\int_{\Omega^{\varepsilon} \cap T} \nabla \vec{u}_{H}: \nabla \vec{v}-\int_{\Omega^{\varepsilon} \cap T} p_{H}^{\prime} \operatorname{div} \vec{v}=\sum_{E \in \mathcal{E}_{H}} \vec{\lambda}_{E}^{T} \cdot \int_{E} \vec{v} \quad \forall \vec{v} \in V(T),
$$

with $\vec{\lambda}_{E}^{T}=\left(\lambda_{E, 1}^{T}, \ldots, \lambda_{E, d}^{T}\right)^{T}$. Integrating by parts converts this into the strong form: for any $T \in \mathcal{T}_{H}$

$$
\begin{aligned}
-\Delta \vec{u}_{H}+\nabla p_{H}^{\prime} & =0 \text { on } \Omega^{\varepsilon} \cap T, \\
\vec{u}_{H} & =0 \text { on } B^{\varepsilon} \cap T, \\
\nabla \vec{u}_{H} n-p_{H}^{\prime} n & =\vec{\lambda}_{E}^{T} \text { on } E \cap \Omega^{\varepsilon} \quad \forall \text { edges } E \text { of } \partial T .
\end{aligned}
$$

We see thus that $\vec{u}_{H} \in V_{H}$ and that $p_{H}^{\prime}$ is uniquely determined by $\vec{u}_{H}$ (indeed, for any $\vec{u}_{H}$ fixed in the formulas above, the gradient $\nabla p_{H}^{\prime}$ is uniquely determined on any triangle by the equation in the first line, and the average $p_{H}^{\prime}$ over any triangle is 0 ).

Copyright (c) by SIAM. Unauthorized reproduction of this article is prohibited. 
We have thus $p_{H}^{\prime}=\pi_{H}\left(\vec{u}_{H}\right)$ with $\vec{u}_{H} \in V_{H}$, which proves that $\left(\vec{u}_{H}, p_{H}\right)$ belongs to the space defined by (3.5).

Reversing the arguments above, we prove easily that any $\left(\vec{u}_{H}, p_{H}\right) \in X_{H}$ in the sense of definition (3.5) satisfies also relation (3.2). We conclude thus that the definitions (3.2) and (3.5) are equivalent.

It remains to prove $X_{H}^{e x t}=X_{H}+X_{H}^{0}$, i.e., that any $(\vec{u}, p) \in X_{H}^{e x t}$ can be represented as

$$
\vec{u}=\vec{u}_{H}+\vec{u}^{0}, \quad p_{H}=\pi_{H}\left(\vec{u}_{H}\right)+\bar{p}_{H}+p^{0},
$$

with some $\vec{u}_{H} \in V_{H}, \vec{u}^{0} \in V_{H}^{0}, \bar{p}_{H} \in M_{H}$, and $p^{0} \in M_{H}^{0}$. This is equivalent to the following statement: for any $(\vec{u}, p) \in X_{H}^{\text {ext }}$ there exists $\left(\vec{u}^{0}, p^{0}\right) \in X_{H}^{0}$ such that

$$
c\left(\left(\vec{u}^{0}, p^{0}\right),(\vec{v}, q)\right)=c((\vec{u}, p),(\vec{v}, q)) \quad \forall(\vec{v}, q) \in X_{H}^{0} .
$$

In order to prove the existence of such $\left(\vec{u}^{0}, p^{0}\right)$, we pick up any triangle $T \in \mathcal{T}_{H}$ and remark that the restriction of $\left(\vec{u}^{0}, p^{0}\right)$ to the triangle $T$ belongs to $V_{\int 0}(T) \times L_{0}^{2}\left(T \cap \Omega^{\varepsilon}\right)$ and satisfies

$$
\begin{gathered}
\int_{T \cap \Omega^{\varepsilon}} \nabla \vec{u}^{0}: \nabla \vec{v}-\int_{T \cap \Omega^{\varepsilon}} p^{0} \operatorname{div} \vec{v}=\int_{T \cap \Omega^{\varepsilon}} \nabla \vec{u}: \nabla \vec{v}-\int_{T \cap \Omega^{\varepsilon}} p \operatorname{div} \vec{v} \quad \forall \vec{v} \in V_{\int 0}(T), \\
\int_{T \cap \Omega^{\varepsilon}} q \operatorname{div} \vec{u}^{0}=\int_{T \cap \Omega^{\varepsilon}} q \operatorname{div} \vec{u} \quad \forall q \in L_{0}^{2}(T) .
\end{gathered}
$$

This is a standard saddle point problem, and its solution exists since we obviously have the inf-sup property

$$
\inf _{q \in L_{0}^{2}\left(T \cap \Omega^{\varepsilon}\right)} \sup _{\vec{v} \in V_{\int 0}(T)} \frac{\int_{T \cap \Omega^{\varepsilon}} q \operatorname{div} \vec{v}}{\|q\|_{L^{2}(T)}|\vec{v}|_{H^{1}(T)}}>0 .
$$

Finally, it is easy to see that $X_{H} \cap X_{H}^{0}=\{0\}$; i.e., the relation (3.1) holds true. Indeed, if $(\vec{u}, p) \in X_{H} \cap X_{H}^{0}$, then

$$
c((\vec{u}, p),(\vec{v}, q))=0
$$

both for $(\vec{v}, q) \in X_{H}$ and for $(\vec{v}, q) \in X_{H}^{0}$. Since $X_{H}^{e x t}=X_{H}+X_{H}^{0}$, we have (3.8) for any $(\vec{v}, q) \in X_{H}^{e x t}$, which implies $(\vec{u}, p)=0$ by the inf-sup property (2.5).

3.2. Basis functions for the space $\boldsymbol{V}_{\boldsymbol{H}}$. The following lemma shows that one can construct a basis for $V_{H}$ consisting of functions associated to the edges of the mesh. Each basis function is supported in the patch $\omega_{E}$ consisting of two triangles adjacent to an edge $E \in \mathcal{E}_{H}$ as in the classical Crouzeix-Raviart FEM.

Lemma 3.2. For any edge $E \in \mathcal{E}_{H}$ one can construct $\vec{\Phi}_{E, i} \in V_{H}, i=1, \ldots, d$, such that $\int_{E} \vec{\Phi}_{E, i}=\vec{e}_{i}$, with $\left\{\vec{e}_{1}, \ldots, \vec{e}_{d}\right\}$ being the canonical basis of $\mathbb{R}^{d}$, and $\int_{E^{\prime}} \vec{\Phi}_{E, i}=$ 0 for all $E^{\prime} \in \mathcal{E}_{H}, E^{\prime} \neq E$. These functions form a basis of $V_{H}$ :

$$
V_{H}=\operatorname{span}\left\{\vec{\Phi}_{E, i}, \quad E \in \mathcal{E}_{H}, i=1, \ldots, d\right\} .
$$

Moreover, $\operatorname{supp}\left(\vec{\Phi}_{E, i}\right) \subset \omega_{E}$, i.e., the ensemble of two triangles from $\mathcal{T}_{H}$ adjacent to the edge $E \in \mathcal{E}_{H}$. 
Proof. For any edge $E \in \mathcal{E}_{H}$ there exist functions ${ }^{2} \vec{v}_{E, i} \in V_{H}^{\text {ext }}, i=1, \ldots, d$, such that $\int_{E} \vec{v}_{E, i}=e_{i}$ and $\int_{E^{\prime}} \vec{v}_{E, i}=0$ for all $E^{\prime} \in \mathcal{E}_{H}, E^{\prime} \neq E$. The space $V_{H}^{\text {ext }}$ can be evidently decomposed as

$$
V_{H}^{e x t}=V_{H}^{0} \oplus \operatorname{span}\left\{\vec{v}_{E, i}, E \in \mathcal{E}_{H}, i=1, \ldots, d\right\} .
$$

We also have the decomposition

$$
V_{H}^{e x t}=V_{H}^{0} \oplus V_{H},
$$

which implies for any $E \in \mathcal{E}_{H}$ and $i=1, \ldots, d$ that there exist functions $\vec{\Phi}_{E, i} \in V_{H}$ and $\vec{v}_{E, i}^{0} \in V_{H}^{0}$ such that

$$
\vec{v}_{E, i}=\vec{v}_{E, i}^{0}+\vec{\Phi}_{E, i}
$$

Thus, for any $\vec{u} \in V_{H}^{e x t}$,

$$
\begin{aligned}
\vec{u} & =\vec{u}^{0}+\sum_{E \in \mathcal{E}_{H}, i=1, \ldots, d} \alpha_{E, i} \vec{v}_{E, i} \\
& =\left[\vec{u}^{0}+\sum_{E \in \mathcal{E}_{H}, i=1, \ldots, d} \alpha_{E, i} \vec{v}_{E, i}^{0}\right]+\sum_{E \in \mathcal{E}_{H}, i=1, \ldots, d} \alpha_{E, i} \vec{\Phi}_{E, i},
\end{aligned}
$$

with some $\vec{u}^{0} \in V_{H}^{0}$ and $\alpha_{E, i} \in \mathbb{R}$. This proves (3.9) since the expression in the brackets in (3.11) represents a function in $V_{H}^{0}$ and we have the direct sum (3.10).

It remains to prove that the support of $\vec{\Phi}_{E, i}$ is indeed within the patch $\omega_{E}$. To this end, consider the function $\vec{\Phi}_{E, i}^{\prime} \in V_{H}^{\text {ext }}$ such that $\vec{\Phi}_{E, i}^{\prime}=\vec{\Phi}_{E, i}$ on $\omega_{E}$ and $\vec{\Phi}_{E, i}^{\prime}=0$ outside $\omega_{E}$. According to definition (3.4), $\vec{\Phi}_{E, i}^{\prime} \in V_{H}$. Moreover, $\vec{\Phi}_{E, i}^{\prime}-\vec{\Phi}_{E, i} \in V_{H}^{0}$, so that $\vec{\Phi}_{E, i}^{\prime}-\vec{\Phi}_{E, i} \in V_{H} \cap V_{H}^{0}=\{0\}$. Thus, $\vec{\Phi}_{E, i}$ coincides with $\vec{\Phi}_{E, i}^{\prime}$, whose support is in $\omega_{E}$ by construction.

Remark 3.3. The explicit construction of the basis functions introduced above is as follows: for any $E \in \mathcal{E}_{H}$ we construct $\vec{\Phi}_{E, i}: \Omega \rightarrow \mathbb{R}^{d}$ and the accompanying pressure $\pi_{E, i}: \Omega^{\varepsilon} \rightarrow \mathbb{R}$ such that $\vec{\Phi}_{E, i}$ and $\pi_{E, i}$ vanish outside the two triangles $T_{1}, T_{2}$ adjacent to $E$ and they solve, on each of these two triangles,

$$
\begin{array}{rlrl}
-\Delta \vec{\Phi}_{E, i}+\nabla \pi_{E, i} & =0 & & \text { on } \Omega^{\varepsilon} \cap T_{k}, \\
\operatorname{div} \vec{\Phi}_{E, i} & =\text { const } & & \text { on } \Omega^{\varepsilon} \cap T_{k}, \\
\vec{\Phi}_{E, i} & =0 & & \text { on } B^{\varepsilon} \cap T_{k}, \\
\nabla \vec{\Phi} n_{E, i}-\pi_{E, i} n & =\text { const } & \text { on } F \cap \Omega^{\varepsilon} & \forall F \in \mathcal{E}\left(T_{k}\right), \\
\int_{F} \vec{\Phi}_{E, i} & =\left\{\begin{array}{ll}
\vec{e}_{i}, F=E & F \neq E \\
0, F \neq E & F \neq
\end{array} \quad \forall F \in \mathcal{E}\left(T_{k}\right),\right. \\
\int_{\Omega^{\varepsilon} \cap T_{k}} \pi_{E, i} & =0 .
\end{array}
$$

\footnotetext{
${ }^{2}$ In fact, these functions are combinations of $\vec{v}_{E, i}^{T}$ and have already been introduced on the previous page. For each edge $E \in \mathcal{E}_{H}$, the new function $\vec{v}_{E, i}$ is supported on two triangles $T_{1}, T_{2}$ sharing $E$ and is equal to $\vec{v}_{E, i}^{T_{k}}$ on $T_{k}, k=1,2$.
}

Copyright $@$ by SIAM. Unauthorized reproduction of this article is prohibited. 
In the weak form this gives the following: find $\vec{\Phi}_{E, i} \in H^{1}\left(T_{k}\right)$ such that $\vec{\Phi}_{E, i}=0$ on $T_{k} \cap B^{\varepsilon}, \pi_{E, i} \in L_{0}^{2}\left(T_{k} \cap \Omega^{\varepsilon}\right)$, and the Lagrange multipliers $\vec{\lambda}_{F}, F \in \mathcal{E}\left(T_{k}\right)$, satisfying

$$
\begin{aligned}
\int_{T_{k} \cap \Omega^{\varepsilon}} \nabla \vec{\Phi}_{E, i}: \nabla \vec{v}-\int_{T_{k} \cap \Omega^{\varepsilon}} \pi_{E, i} \operatorname{div} \vec{v}+\sum_{F \in \mathcal{E}\left(T_{k}\right)} \vec{\lambda}_{F} \cdot \int_{F} \vec{v}=0 \\
\forall \vec{v} \in H^{1}\left(T_{k}\right) \text { such that } \vec{v}=0 \text { on } T_{k} \cap B^{\varepsilon}, \\
\int_{T_{k} \cap \Omega^{\varepsilon}} q \operatorname{div} \vec{\Phi}_{E, i}=0 \quad \forall q \in L_{0}^{2}\left(T_{k} \cap \Omega^{\varepsilon}\right), \\
\sum_{F \in \mathcal{E}\left(T_{k}\right)} \vec{\mu}_{F} \cdot \int_{F} \vec{\Phi}_{E, i}=\vec{\mu}_{E} \cdot \vec{e}_{i} \forall \vec{\mu}_{F} \in \mathbb{R}^{d}, F \in \mathcal{E}\left(T_{k}\right) .
\end{aligned}
$$

We note that $L_{0}^{2}\left(T_{k} \cap \Omega^{\varepsilon}\right)=\left\{q \in L^{2}\left(T_{k} \cap \Omega^{\varepsilon}\right): \int_{\Omega^{\varepsilon} \cap T_{k}} q=0\right\}$. The explicit formula for the operator $\pi_{H}$ then reads

$$
\pi_{H}\left(\sum_{E, i} u_{E, i} \vec{\Phi}_{E, i}\right)=\sum_{E, i} u_{E, i} \pi_{E, i} .
$$

Remark 3.4. The space $V_{H}$ is reduced to the classical Crouzeix-Raviart finite element space in the case without holes $B^{\varepsilon}=\varnothing$. Indeed, it is easy to see that the basis functions constructed above can be written in this case as $\vec{\Phi}_{E, i}=\Phi_{E} \vec{e}_{i}$, where $\Phi_{E}$ is linear on any triangle $T \in \mathcal{T}_{H}$, discontinuous across the edges, and such that $\int_{E} \Phi_{E}=1$ and $\int_{E^{\prime}} \Phi_{E}=0$ for all $E^{\prime} \in \mathcal{E}_{H}, E^{\prime} \neq E$.

3.3. Crouzeix-Raviart MsFEM coarse solution. We now define the MsFEM solution to problem $(2.1)-(2.2)$ as $\left(\vec{u}_{H}, p_{H}\right) \in X_{H}$ such that

$$
c\left(\left(\vec{u}_{H}, p_{H}\right),\left(\vec{v}_{H}, q_{H}\right)\right)=\left(f, \vec{v}_{h}\right) \quad \forall\left(\vec{v}_{H}, q_{H}\right) \in X_{H} .
$$

We note that $\vec{u}_{H}, p_{H}$ can be represented as $\vec{u}_{H} \in V_{H}$ and $p_{H}=\pi_{H}\left(\vec{u}_{H}\right)+\bar{p}_{H}$ with $\bar{p}_{H} \in M_{H}$. We have also $\left(\pi_{H}\left(\vec{u}_{H}\right), \operatorname{div} \vec{v}_{H}\right)=0$ for all $\vec{u}_{H}, \vec{v}_{H} \in V_{H}$. Hence, the problem above can be recast as follows: find $\vec{u}_{H} \in V_{H}$ and $\bar{p}_{H} \in M_{H}$ such that

$$
\begin{gathered}
\int_{\Omega^{\varepsilon}} \nabla \vec{u}_{H}: \nabla \vec{v}_{H}-\int_{\Omega^{\varepsilon}} \bar{p}_{H} \operatorname{div} \vec{v}_{H}=\int_{\Omega^{\varepsilon}} \vec{f} \cdot \vec{v}_{H} \quad \forall \vec{v}_{H} \in V_{H}, \\
\int_{\Omega^{\varepsilon}} \bar{q}_{H} \operatorname{div} \vec{u}_{H}=0 \quad \forall \bar{q}_{H} \in M_{H} .
\end{gathered}
$$

We note that the second equation in the system above entails $\operatorname{div} \vec{u}_{H}=0$ on $\Omega^{\varepsilon}$ since $\operatorname{div} V_{H}=M_{H}$. We can thus eliminate the pressure from this system by introducing the subspace of $V_{H}$ consisting of divergence free functions: denote

$$
Z_{H}=\left\{\vec{v}_{H} \in V_{H} \text { such that } \operatorname{div} \vec{v}_{H}=0\right\} .
$$

The discrete velocity solution can then be alternatively defined as $\vec{u}_{H} \in Z_{H}$ such that

$$
\int_{\Omega^{\varepsilon}} \nabla \vec{u}_{H}: \nabla \vec{v}_{H}=\int_{\Omega^{\varepsilon}} \vec{f} \cdot \vec{v}_{H} \quad \forall \vec{v}_{H} \in Z_{H}
$$

This ensures immediately existence and uniqueness of $\vec{u}_{H}$. Existence and uniqueness of $p_{H}$ now follow from the fact $\operatorname{div} V_{H}=M_{H}$.

Copyright $@$ by SIAM. Unauthorized reproduction of this article is prohibited. 
3.4. The inf-sup property for the spaces $V_{H}$ and $M_{H}$. According to [30, Corollary 2.4],

$$
\forall p \in M \exists \vec{v} \in V \text { such that } \operatorname{div} \vec{v}=p \text { and }\|\vec{v}\|_{V} \leq \frac{1}{\gamma}\|p\|_{M},
$$

with a constant $\gamma>0$ (depending on $\Omega^{\varepsilon}$ ). This implies in particular the inf-sup condition (2.4). We shall now prove that this condition remains valid on the discrete level.

Lemma 3.5. There exists a mesh independent constant $\gamma>0$ (in fact, $\gamma$ can be taken the same as in (3.13)) such that

$$
\inf _{p_{H} \in M_{H}} \sup _{\vec{v}_{H} \in V_{H}} \frac{\int_{\Omega^{\varepsilon}} p_{H} \operatorname{div} \vec{v}_{H}}{\left\|p_{H}\right\|_{M}\left\|\vec{v}_{H}\right\|_{V}} \geq \gamma .
$$

Proof. Take any $p_{H} \in M_{H}$ and construct $\vec{v} \in V$ as in (3.13), i.e.,

$$
\operatorname{div} \vec{v}=p_{H} \text { on } \Omega \text { and }\|\vec{v}\|_{V} \leq \frac{1}{\gamma}\left\|p_{H}\right\|_{M} .
$$

Decompose $\vec{v}=\vec{v}_{H}+\vec{v}^{0}$ with $\vec{v}_{H} \in V_{H}$ and $\vec{v}^{0} \in V_{H}^{0}$. Observe that $p_{H}=$ const and $\operatorname{div} \vec{v}_{H}=$ const on $T \cap \Omega^{\epsilon}$. This means that $\operatorname{div} \vec{v}^{0}=$ const on $T \cap \Omega^{\epsilon}$. On the other hand,

$$
\int_{T \cap \Omega^{\epsilon}} \operatorname{div} \vec{v}^{0}=\int_{\partial T \cap \Omega^{\epsilon}} \vec{v}^{0} \cdot n=0,
$$

and hence $\operatorname{div} \vec{v}^{0}=0$. By construction of $V_{H}$ in Lemma 3.1,

$$
0=c\left(\left(\vec{v}_{H}, \pi_{H}\left(\vec{v}_{H}\right)\right),\left(\vec{v}^{0}, 0\right)\right)=\int_{\Omega^{\varepsilon}} \nabla \vec{v}_{H}: \nabla \vec{v}^{0} ;
$$

i.e., $\vec{v}_{H}$ and $\vec{v}^{0}$ are orthogonal in the scalar product of space $V$. Now, by Pythagoras' theorem,

$$
\left\|\vec{v}_{H}\right\|_{V} \leq\left\|\vec{v}_{H}+\vec{v}^{0}\right\|_{V}=\|\vec{v}\|_{V} \leq \frac{1}{\gamma}\left\|p_{H}\right\|_{M} .
$$

Noting that $\operatorname{div} \vec{v}_{H}=p_{H}$, this gives (3.14).

\subsection{Some error estimates.}

Lemma 3.6. Let $(\vec{u}, p) \in X$ be the solution to (2.1)-(2.2), and let $\left(\vec{u}_{H}, p_{H}\right) \in X_{H}$ be the discrete solution defined by (3.12). Then

$$
\begin{aligned}
&\left|\vec{u}-\vec{u}_{H}\right|_{H^{1}}+\gamma\left\|\Pi_{H} p-\bar{p}_{H}\right\|_{L^{2}} \\
& \leq C H \|\left.\vec{f}\right|_{L^{2}}+C H^{\frac{1}{2}} \inf _{\vec{c}_{E} \in \mathbb{R}^{2}, E \in \mathcal{E}_{H}}\left(\sum_{E \in \mathcal{E}_{H}}\left\|\nabla \vec{u} n-p n-\vec{c}_{E}\right\|_{L^{2}(E)}^{2}\right)^{\frac{1}{2}},
\end{aligned}
$$

where $C>0$ is a constant depending only on the regularity of the mesh, $\gamma$ is the inf-sup constant from (3.13), $\Pi_{H}$ is the $L^{2}$-orthogonal projector on $M_{H}$, and $\bar{p}_{H}$ is a piecewise constant part of $p_{H}$, i.e., $p_{H}=\pi_{H}\left(\vec{u}_{H}\right)+\bar{p}_{H}$.

Proof. As usual, to prove an a priori error estimate we are going to first construct an interpolant of the exact solution $(\vec{u}, p) \in X$ and then invoke a (slightly generalized)

Copyright (c) by SIAM. Unauthorized reproduction of this article is prohibited. 
version of Céa's lemma. A good interpolant $\left(\vec{u}_{I}, p_{I}\right) \in X_{H}$ may be introduced by requiring

$$
(\vec{u}, p)=\left(\vec{u}_{I}, p_{I}\right)+\left(\vec{u}^{0}, p^{0}\right),
$$

with $\left(\vec{u}^{0}, p^{0}\right) \in X_{H}^{0}$. Such a unique decomposition of $(\vec{u}, p)$ exists and is unique due to the decomposition of the space $X_{H}^{e x t}$; cf. (3.1). It is easy to see that $\operatorname{div} \vec{u}^{0}=0$. Indeed, for all $q_{0} \in M_{H}^{0}$,

$$
c\left(\left(\vec{u}^{0}, p^{0}\right),\left(0, q^{0}\right)\right)=c\left((\vec{u}, p),\left(0, q^{0}\right)\right),
$$

i.e.,

$$
\sum_{T \in \mathcal{T}_{H}} \int_{T \cap \Omega^{\varepsilon}} q^{0} \operatorname{div} \vec{u}^{0}=0
$$

since $\operatorname{div} \vec{u}=0$. This implies $\operatorname{div} \vec{u}^{0}=c_{T}=$ const on $T \cap \Omega^{\varepsilon}$ for all $T \in \mathcal{T}_{H}$, but $\int_{\partial T} \vec{u}^{0} \cdot n=0$ due to the definition of $X_{H}^{0}$ so that $c_{T}=0$.

Putting $\operatorname{div} \vec{u}^{0}=0$ into the definition of the bilinear form $c$, we have

$$
\begin{aligned}
\left\|\vec{u}-\vec{u}_{I}\right\|_{V}^{2} & =\left\|\vec{u}^{0}\right\|_{V}^{2}=c\left(\left(\vec{u}^{0}, p^{0}\right),\left(\vec{u}^{0}, p^{0}\right)\right) \\
& =\sum_{T \in \mathcal{T}_{H}} \int_{T \cap \Omega^{\varepsilon}}\left(-\Delta \vec{u}^{0}+\nabla p^{0}\right) \cdot \vec{u}^{0}+\sum_{T \in \mathcal{T}_{H}} \int_{\partial T \cap \Omega^{\varepsilon}}\left(\nabla \vec{u}^{0} n-p^{0} n\right) \cdot \vec{u}^{0} \\
& =\sum_{T \in \mathcal{T}_{H}} \int_{T \cap \Omega^{\varepsilon}} \vec{f} \cdot \vec{u}^{0}+\sum_{T \in \mathcal{T}_{H}} \sum_{E \in \mathcal{E}(T)} \int_{E \cap \Omega^{\varepsilon}}\left(\nabla \vec{u} n-p n-\vec{c}_{E}\right) \cdot \vec{u}^{0}
\end{aligned}
$$

since on each triangle $T \in \mathcal{T}_{H}$,

$$
-\Delta \vec{u}^{0}+\nabla p^{0}=(-\Delta \vec{u}+\nabla p)-\left(-\Delta \vec{u}_{I}+\nabla p_{I}\right)=\vec{f},
$$

and, moreover, on each edge $E \in \mathcal{E}_{H}$,

$$
\begin{aligned}
\int_{E \cap \Omega^{\varepsilon}}\left(\nabla \vec{u}^{0} n-p^{0} n\right) \cdot \vec{v}^{0} & =\int_{E \cap \Omega^{\varepsilon}}(\nabla \vec{u} n-p n) \cdot \vec{v}^{0}-\int_{E \cap \Omega^{\varepsilon}}\left(\nabla \vec{u}_{I} n-p_{I} n\right) \cdot \vec{v}^{0} \\
& =\int_{E \cap \Omega^{\varepsilon}}(\nabla \vec{u} n-p n) \cdot \vec{v}^{0}-\vec{\lambda}_{E} \cdot \int_{E \cap \Omega^{\varepsilon}} \vec{v}^{0} \\
& =\int_{E \cap \Omega^{\varepsilon}}\left(\nabla \vec{u} n-p n-\vec{c}_{E}\right) \cdot \vec{v}^{0}
\end{aligned}
$$

with some constant $\vec{\lambda}_{E}$ depending on $\left(\vec{u}_{I}, p_{I}\right) \in X_{H}$ (cf. definition (3.4) of $V_{H}$ ) and an arbitrary constant $\vec{c}_{E}$ (cf. the definition of $X_{H}^{0}$ ). We now note the Poincaré type inequality

$$
\left\|\vec{v}^{0}\right\|_{L^{2}(T)} \leq C H\left|\vec{v}^{0}\right|_{H^{1}(T)}
$$

and the trace inequality

$$
\left\|\vec{v}^{0}\right\|_{L^{2}(\partial T)} \leq C H^{\frac{1}{2}}\left|\vec{v}^{0}\right|_{H^{1}(T)}
$$

Copyright $@$ ㅇ by SIAM. Unauthorized reproduction of this article is prohibited. 
which are valid since the average $\vec{v}^{0}$ on the edges composing $\partial T$ is zero; see [27] for more details. This allows us to conclude that

$$
\begin{aligned}
\left\|\vec{u}-\vec{u}_{I}\right\|_{V}^{2} & \leq \sum_{T \in \mathcal{T}_{H}}\|\vec{f}\|_{L^{2}(T)}\left\|\vec{u}^{0}\right\|_{L^{2}(T)} \\
& +\sum_{T \in \mathcal{T}_{H}}\left(\sum_{E \in \mathcal{E}(T)}\left\|\nabla \vec{u} n-p n-\vec{c}_{E}\right\|_{L^{2}(E)}^{2}\right)^{\frac{1}{2}}\left\|\vec{u}^{0}\right\|_{L^{2}(\partial T)} \\
& \leq C H\|\vec{f}\|_{L^{2}(\Omega)}\left|\vec{u}^{0}\right|_{H^{1}(\Omega)} \\
& +C H^{\frac{1}{2}}\left(\sum_{E \in \mathcal{E}_{H}}\left\|\nabla \vec{u} n-p n-\vec{c}_{E}\right\|_{L^{2}(E)}^{2}\right)^{\frac{1}{2}}\left|\vec{u}^{0}\right|_{H^{1}(\Omega)} .
\end{aligned}
$$

Finally,

$$
\left\|\vec{u}-\vec{u}_{I}\right\|_{V} \leq C H\|\vec{f}\|_{L^{2}(\Omega)}+C H^{\frac{1}{2}}\left(\sum_{E \in \mathcal{E}_{H}}\left\|\nabla \vec{u} n-p n-\vec{c}_{E}\right\|_{L^{2}(E)}^{2}\right)^{\frac{1}{2}} .
$$

Now we use the $c$-orthogonality between $\left(\vec{u}_{I}, p_{I}\right)$ and $\left(\vec{u}^{0}, p^{0}\right)$ to write, for any $\left(\vec{v}_{H}, q_{H}\right) \in X_{H}$,

$c\left(\left(\vec{u}_{I}, p_{I}\right),\left(\vec{v}_{H}, q_{H}\right)\right)=c\left((\vec{u}, p),\left(\vec{v}_{H}, q_{H}\right)\right)=\int_{\Omega^{\varepsilon}} \vec{f} \cdot \vec{v}_{H}+\sum_{T \in \mathcal{T}_{H}} \int_{\partial T \cap \Omega^{\varepsilon}}(\nabla \vec{u} n-p n) \cdot \vec{v}_{H}$.

Comparing it with (3.12), we conclude that

$$
c\left(\left(\vec{u}_{I}-\vec{u}_{H}, p_{I}-p_{H}\right),\left(\vec{v}_{H}, q_{H}\right)\right)=\sum_{T \in \mathcal{T}_{H}} \int_{\partial T \cap \Omega^{\varepsilon}}(\nabla \vec{u} n-p n) \cdot \vec{v}_{H} \quad \forall\left(\vec{v}_{H}, q_{H}\right) \in X_{H} .
$$

For any $\vec{v} \in X_{H}^{\text {ext }}$ denote by $\{\vec{v}\}_{E}$ the average of $\vec{v}$ over an edge $E$. We can then rewrite the sum of boundary integrals as

$$
\sum_{T \in \mathcal{T}_{H}} \int_{\partial T \cap \Omega^{\varepsilon}}(\nabla \vec{u} n-p n) \cdot \vec{v}_{H}=\sum_{T \in \mathcal{T}_{H}} \sum_{E \in \mathcal{E}(T)} \int_{E \cap \Omega^{\varepsilon}}(\nabla \vec{u} n-p n) \cdot\left(\vec{v}_{H}-\left\{\vec{v}_{H}\right\}_{E}\right) .
$$

Indeed, any edge $E$ comes into these sums two times as contributions from the two adjacent triangles, and thus the additions with $\left\{\vec{v}_{H}\right\}_{E}$ cancel each other. We can now proceed by inserting an arbitrary constant $\vec{c}_{E}$ on each edge:

$$
\sum_{T \in \mathcal{T}_{H}} \int_{\partial T \cap \Omega^{\varepsilon}}(\nabla \vec{u} n-p n) \cdot \vec{v}_{H}=\sum_{T \in \mathcal{T}_{H}} \sum_{E \in \mathcal{E}(T)} \int_{E \cap \Omega^{\varepsilon}}\left(\nabla \vec{u} n-p n-\vec{c}_{E}\right) \cdot\left(\vec{v}_{H}-\left\{\vec{v}_{H}\right\}_{E}\right) .
$$

Finally, reusing the Poincaré type inequality (3.16) and the trace inequality (3.17), we conclude in a similar way as above that

$$
c\left(\left(\vec{u}_{I}-\vec{u}_{H}, p_{I}-p_{H}\right),\left(\vec{v}_{H}, q_{H}\right)\right) \leq C H^{\frac{1}{2}}\left(\sum_{E \in \mathcal{E}_{H}}|| \nabla \vec{u} n-p n-\vec{c}_{E} \|_{L^{2}(E)}^{2}\right)^{\frac{1}{2}}\left|\vec{v}_{H}\right|_{H^{1}(\Omega)}
$$

for any $\left(\vec{v}_{H}, q_{H}\right) \in X_{H}$. 
Taking $\vec{v}_{H}=\vec{u}_{I}-\vec{u}_{H}, q_{H}=\pi_{H}\left(\vec{v}_{H}\right)$ and noting $\operatorname{div} \vec{v}_{H}=0$ yields

$$
\left\|\vec{u}_{I}-\vec{u}_{H}\right\|_{V} \leq C H^{\frac{1}{2}}\left(\sum_{E \in \mathcal{E}_{H}}\left\|\nabla \vec{u} n-p n-\vec{c}_{E}\right\|_{L^{2}(E)}^{2}\right)^{\frac{1}{2}} .
$$

Combining the estimates (3.18) and (3.20) with the triangle inequality yields

$$
\left\|\vec{u}-\vec{u}_{H}\right\|_{V} \leq C H\|\vec{f}\|_{L^{2}(\Omega)}+C H^{\frac{1}{2}}\left(\sum_{E \in \mathcal{E}_{H}}\left\|\nabla \vec{u} n-p n-\vec{c}_{E}\right\|_{L^{2}(E)}^{2}\right)^{\frac{1}{2}},
$$

which is the announced error estimate for the velocity.

In order to get the error estimate for the pressure, we rewrite (3.19) for arbitrary $\vec{v}_{H} \in V_{H}$ and $q_{H}=\pi_{H}\left(\vec{v}_{H}\right)$ as

$$
\begin{aligned}
& -\int_{\Omega^{\epsilon}}\left(p_{I}-p_{H}\right) \operatorname{div} \vec{v}_{H}=-\int_{\Omega^{\epsilon}}\left(\Pi_{H} p-\bar{p}_{H}\right) \operatorname{div} \vec{v}_{H} \\
& \leq-\int_{\Omega^{\epsilon}} \nabla\left(\vec{u}_{I}-\vec{u}_{H}\right): \nabla \vec{v}_{H}+C H^{\frac{1}{2}}\left(\sum_{E \in \mathcal{E}_{H}}|| \nabla \vec{u} n-p n-\vec{c}_{E} \|_{L^{2}(E)}^{2}\right)^{\frac{1}{2}}\left|\vec{v}_{H}\right|_{H^{1}(\Omega) .}
\end{aligned}
$$

We have used here $p_{I}=\Pi_{H} p, \bar{p}_{H}=\Pi_{H} p_{H}$ and the fact that $\operatorname{div} \vec{v}_{H} \in M_{H}$. It remains to employ the inf-sup property (3.14) and the already proven error estimate for the velocity. This gives the announced bound for $\left\|\Pi_{H} p-\bar{p}_{H}\right\|_{M}$.

The error estimate of the preceding lemma is not completely satisfactory because of the oscillating nature of the exact solution, which can make the norms on the edges quite big. A complete error estimate in the case of the Poisson equation and periodically placed holes is available in [28]. A similar result will be proved in the forthcoming paper [32].

4. Implementation issues. The Crouzeix-Raviart MsFEM as presented so far is not directly implementable since it relies on the exact solutions of the local problems in the construction of the basis functions; cf. Remark 3.3. In practice, these problems should be discretized on mesh sufficiently fine to resolve the geometry of obstacles. For highly nonperiodic pattern of obstacles, complicated body-fitted unstructured mesh is likely what engineers would resort to. In this paper, we opt for performing our computations on a simple uniform Cartesian grid using the penalization method. We shall explain first how to do it when solving the original problem (2.1)-(2.2) as a whole without resorting to the MsFEM technique (this is needed anyway to construct the reference solutions in our numerical experiments).

4.1. Application of the penalization method to compute the reference solution. To avoid complex and ad hoc grid generation methods when solving problem (2.1)-(2.2) in $\Omega^{\epsilon}$ we replace it with the penalized problem

$$
\begin{array}{rll}
-\nabla \cdot\left(\nu^{\kappa} \nabla \vec{u}\right)+\sigma^{\kappa} \vec{u}+\nabla p=\vec{f}^{\kappa} & \text { in } & \Omega, \\
\nabla \cdot \vec{u}=0 & \text { in } & \Omega, \\
\vec{u}=\vec{w} & \text { on } & \partial \Omega,
\end{array}
$$

in which

$$
\nu^{\kappa}=\left\{\begin{array}{ll}
\frac{1}{h} & \text { in } B^{\epsilon}, \\
\nu & \text { in } \Omega^{\epsilon},
\end{array} \quad \sigma^{\kappa}=\left\{\begin{array}{rl}
\frac{1}{h^{3}} & \text { in } B^{\epsilon}, \\
0 & \text { in } \Omega^{\epsilon},
\end{array} \quad f^{\kappa}= \begin{cases}0 & \text { in } B^{\epsilon}, \\
f & \text { in } \Omega^{\epsilon} .\end{cases}\right.\right.
$$


Here $h$ is the size of the fine scale mesh element used to capture a highly oscillatory solution. The penalization coefficients $\nu^{\kappa}$ and $\sigma^{\kappa}$ force the solution to vanish rapidly inside the obstacles; their values are chosen such that the optimal approximations are obtained. Other variants of penalization methods were studied in [33], and a test for a range of values for $\nu^{\kappa}$ and $\sigma^{\kappa}$ has been discussed in [34].

To calculate the reference solutions, we utilize the Q1-Q1 FEM, which has velocity and pressure degrees of freedom defined at the same set of grid points, namely the uniform Cartesian grid $\mathcal{T}_{h}$ of step $h$. As is well known (cf. [35]), this choice of velocity and pressure spaces requires some stabilization, which weakens the condition $\nabla \cdot \vec{u}=0$. The simplest way to achieve this is by perturbing the incompressibility constraint with a pressure Laplacian term; see [36]. Our numerical problem thus reduces to finding $\vec{u}_{h}$ and $p_{h}$ from the Q1-Q1 finite element space such that

$$
\begin{gathered}
\int_{\Omega} \nu^{\kappa} \nabla \vec{u}_{h}: \nabla \vec{v}_{h}+\int_{\Omega} \sigma^{\kappa} \vec{u}_{h} \cdot \vec{v}_{h}-\int_{\Omega} p_{h} \nabla \cdot \vec{v}_{h}=\int_{\Omega} \vec{v}_{h} \cdot \vec{f} \quad \forall \vec{v}_{h}, \\
-\int_{\Omega} q_{h} \nabla \cdot \vec{u}_{h}-\theta h^{2} \int_{\Omega} \nabla p_{h} \cdot \nabla q_{h}=0 \quad \forall q_{h},
\end{gathered}
$$

where $\theta>0$ is the stabilization parameter and $\vec{v}_{h}, q_{h}$ go over the same finite element space. Throughout our simulation, $\theta=0.01$ is used [37].

4.2. Calculation of Crouzeix-Raviart MsFEM basis functions. The calculations of the multiscale basis in this paper are carried out similarly to that of the reference solution. We introduce the fine meshes $\mathcal{T}_{h}(T)$ for each element $T$ of the coarse mesh $\mathcal{T}_{H}$ (in all of our numerical experiments these local meshes will be just submeshes of the global fine mesh $\mathcal{T}_{h}$ ). The MsFEM basis functions are then computed as follows: for any $E \in \mathcal{E}_{H}$ we construct $\vec{\Phi}_{E, i}$ and the accompanying pressure $\pi_{E, i}$ supported in the two triangles $T_{1}, T_{2}$ adjacent to $E$ and belonging to the Q1-Q1 FEM spaces on the meshes $\mathcal{T}_{h}\left(T_{1}\right)$ and $\mathcal{T}_{h}\left(T_{2}\right)$. On each of these two triangles, they solve

$$
\begin{array}{r}
\int_{T_{k}} \nu^{\kappa} \nabla \vec{\Phi}_{E, i}: \nabla \vec{v}_{h}+\int_{T_{k}} \sigma^{\kappa} \vec{\Phi}_{E, i} \cdot \vec{v}_{h}-\int_{T_{k}} \pi_{E, i} \nabla \cdot \vec{v}_{h}+\sum_{F \in \mathcal{E}\left(T_{k}\right)} \vec{\lambda}_{F} \cdot \int_{F} \vec{\Phi}_{E, i}=0 \quad \forall \vec{v}_{h}, \\
-\int_{\Omega} q_{h} \nabla \cdot \vec{\Phi}_{E, i}-\theta h^{2} \int_{\Omega} \nabla \pi_{E, i} \cdot \nabla q_{h}=0 \quad \forall q_{h}, \\
\sum_{F \in \mathcal{E}\left(T_{k}\right)} \vec{\mu}_{F} \cdot \int_{F} \vec{\Phi}_{E, i}=\vec{\mu}_{E} \cdot \vec{e}_{i} \\
\forall \vec{\mu}_{F} \in \mathbb{R}^{d}, F \in \mathcal{E}\left(T_{k}\right),
\end{array}
$$

where $\vec{v}_{h}$ and $q_{h}$ go over the same finite element spaces as $\vec{\Phi}_{E, i}$ and $\pi_{E, i}$. We also recall that $\mathcal{E}\left(T_{k}\right)$ denotes the set of all the edges of the triangle $T_{k}$ and that $\theta>0$ is the stabilization parameter.

4.3. Note on boundary condition. Special treatment of the boundary condition is applied; i.e., we approximate the strong form of the nonhomogeneous Dirichlet boundary condition with

$$
\int_{E} \vec{u}_{H}=\int_{E} \vec{w} \quad \forall E \in \mathcal{E}_{H} \text { on } \partial \Omega .
$$

This, together with the construction of the basis functions in Remark 3.3, yields the formula for the boundary edge contributions in the expansion of the MsFEM 


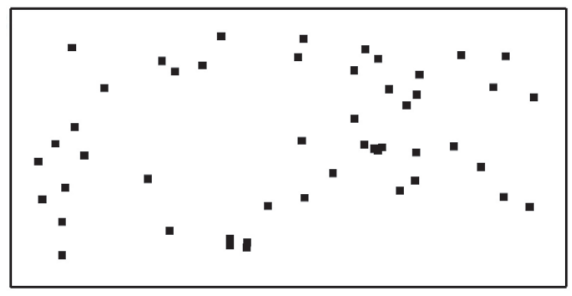

FIG. 2. The computational domain with 49 arbitrarily placed obstacles for the cavity flow test.

solution with respect to the basis functions $\vec{u}_{H}=\sum_{E, i} u_{E, i} \Phi_{E, i}$ : for any $E \in \mathcal{E}_{H}$ on $\partial \Omega$, one puts

$$
u_{E, i}=\int_{E} \vec{w} \cdot \vec{e}_{i} .
$$

This approach is a modification with respect to the earlier works [27], [28], where the boundary conditions were strongly incorporated into the definition of $V_{H}$. Our approach therefore gives more flexibility when implementing nonzero $g$. It will be demonstrated in the later sections how the application of this approach on our MsFEM gives satisfactory results.

5. Numerical results. Homogeneous $\nu=1$ is assumed throughout our tests. The heterogeneities in the problems are represented by sporadic placements of obstacles. However, the application of oscillating $\nu$ is straightforward. In this paper, reconstruction of a fine scale pressure field is not emphasized. In any case, the coarse scale pressure field (element-wise constant) can always be recovered.

5.1. Enclosed flows in heterogeneous media. In the first example, we consider the cavity flow problem. In this problem, all the velocities are known at $\partial \Omega$ (enclosed flow) in which the pressure is unique only up to a constant. The velocities vanish everywhere at boundaries except at the top of the domain $\Omega=[-1 \leq x \leq$ $1,0 \leq y \leq 1]$, where the tangential velocity is set to be $u_{x}=1$. This tangential velocity is the only force that drives the flow. Forty-nine small obstacles, each with a width of $\epsilon=0.0285$, are randomly laid within the cavity; see Figure 2. The reference to which our solutions will be compared is obtained using Q1-Q1 FEM on $640 \times 1280$ elements. In Figures 3 and 4, the solutions of Stokes flow inside the cavity in terms of $u_{x}$ and $u_{y}$ contours using several mesh configurations are given alongside that of the reference solution. Solutions on $32 \times 64$ and $64 \times 128$ elements are almost identical to the reference solution computed on $640 \times 1280$ elements, but in some engineering circumstances, $16 \times 32$ elements already provide quantitatively sufficient measures of the flow.

In Figure 5, the streamlines and the velocity magnitude contours solved using $32 \times 64$ elements are compared with those of the reference. We notice that important recirculation zones at the top half of the domain can be well captured. In this problem, discontinuities are present on both ends of the top lid, and it is shown in Table 1 that our treatment of boundary conditions is sufficient in providing satisfactory solutions. We describe the errors in terms of error norms $L^{1}$ relative, $L^{2}$ relative, and $H^{1}$ relative.

5.2. Open-channel flows in heterogeneous media. Unlike the example of an enclosed flow given above, in the second example we consider open-channel flows. A two-dimensional channel domain $\Omega=[0 \leq x \leq 4,-1 \leq y \leq 1]$ (see Figure 6(a),(b)) 

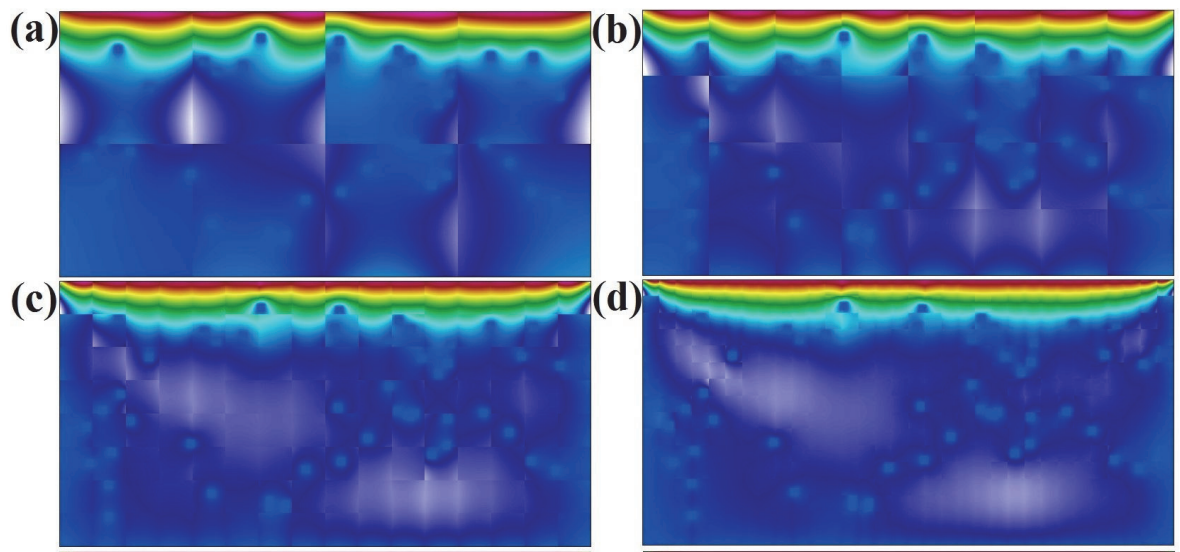

(e)

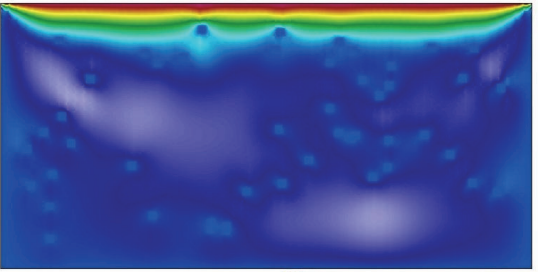

(f)

(g)

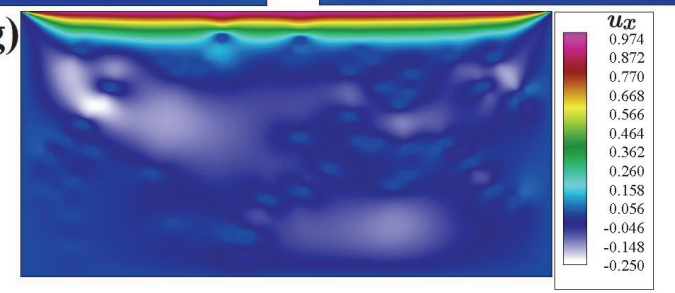

FIG. 3. The plots of velocity $u_{x}$ contours of a cavity flow computed on (a) $2 \times 4$, (b) $4 \times 8$, (c) $8 \times 16$, (d) $16 \times 32$, (e) $32 \times 64$, (f) $64 \times 128$ coarse elements, and the $(\mathrm{g})$ reference solution computed on $640 \times 1280$ elements, all with 49 arbitrarily placed obstacles.

is defined. The first part of this test (Case A) includes 16 obstacles, each with a width of $\epsilon=0.02$. The second part (Case B) includes 144 very fine obstacles, each with a width $\epsilon=0.00832$, all randomly laid in the center of $\Omega$. The parabolic inflow boundary condition, $u_{x}=1-y^{2} ; u_{y}=0$, is taken at the inlet (left), whereas the natural boundary condition $\partial u / \partial n=0$ is assumed at the outlet (right). At the top and bottom walls, no slip boundary conditions $\vec{u}=0$ are taken. In Figures 7 and 8 , Case A solutions of our method on several mesh configurations in terms of $u_{x}$ and $u_{y}$ contours are given alongside those of the reference. As in the cavity flow example, the reference solution is computed on $640 \times 1280$ elements, each with a width of $h=0.003125$. As shown in the example of cavity flow, our results show converging behavior toward reference solutions. Most of the flow features can be quantitatively obtained using a mere $16 \times 32$ elements. In Table 2 , we look at the numerical study of convergence of this problem. In Figure 9, we can see the comparison of streamline and velocity magnitude of the solution on $32 \times 64$ compared to that of the reference. In Figure 10, the pressure reconstruction for several mesh configurations for this case is given and shown to be converging toward the reference solution, where the $L_{2}$ relative error for configuration $128 \times 64$ is 0.141 . 


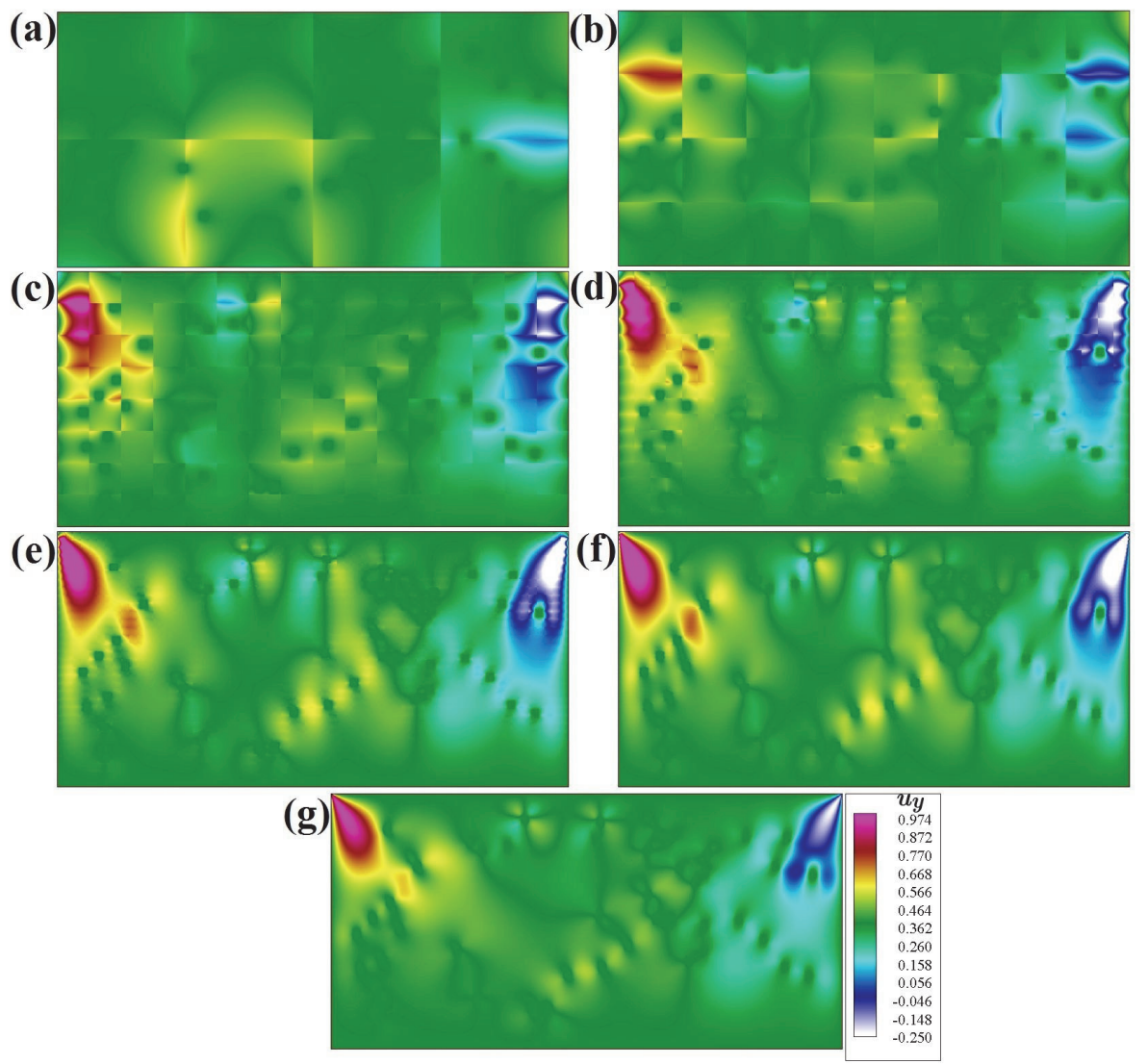

FIG. 4. The plots of velocity $u_{y}$ contours of a cavity flow computed on (a) $2 \times 4$, (b) $4 \times 8$, (c) $8 \times 16$, (d) $16 \times 32$, (e) $32 \times 64$, (f) $64 \times 128$ coarse elements, and the (g) reference solution computed on $640 \times 1280$ elements, all with 49 arbitrarily placed obstacles.

For Case B, Figures 11 and 12 show the contours of $u_{x}$ and $u_{y}$ in comparison to the reference solution, also computed on $640 \times 1280$ elements, each with a width of $h=0.003125$. It is shown that the method is able to recover the presence of very fine obstacles and important flow features already at $16 \times 32$. In Figure 13, the streamlines computed on a $32 \times 64$ coarse mesh are compared with those of the reference on top of the velocity magnitude contour and are in good agreement. The error study of Case $\mathrm{B}$ is shown in Table 3, which also shows converging results as exhibited in other cases even at considerably much larger ratios $(H / \epsilon)$.

In Figures 14 and 15 we illustrate the Crouzeix-Raviart MsFEM basis function $\vec{\Phi}(e, 1)$ when obstacles are both absent and present. We can see that the weak conformity of the Crouzeix-Raviart basis function leads to a natural boundary condition at the coarse element edges, which adapts well with the arbitrary pattern of obstacles. 


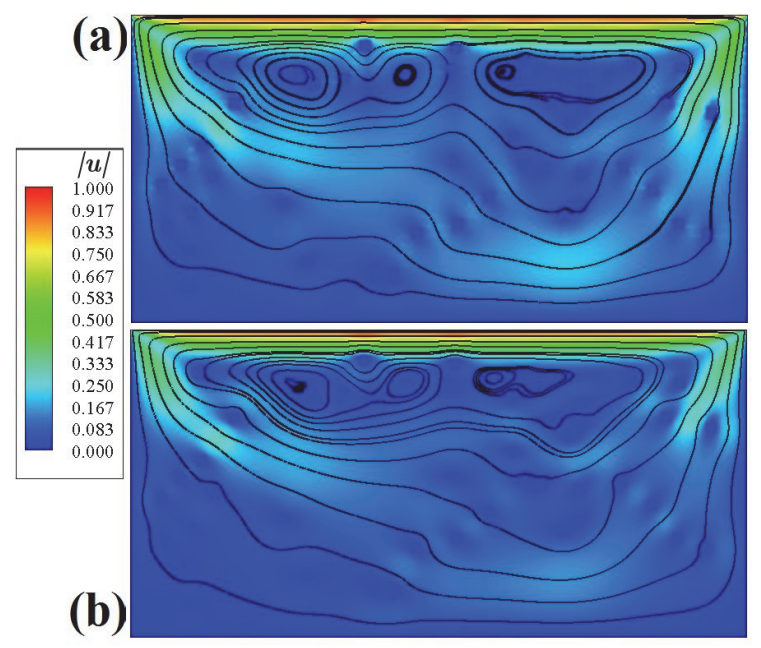

FIG. 5. The plots of velocity magnitude $|u|$ contours of a cavity flow with the streamlines computed using Crouzeix-Raviart MsFEM computed on (a) $32 \times 64$ elements and (b) the reference solution.

TABLE 1

The numerical study of convergence of a cavity flow.

\begin{tabular}{lccccc}
\hline \hline Config. & $(H / \epsilon)$ & $L^{\perp}$ & $L^{2}$ & $H^{\perp}$ & $L^{2} \mathrm{P}$ \\
& Ratio & Relative error & Relative error & Relative error & Relative error \\
\hline $2 \times 4$ & 17.544 & 0.756 & 0.640 & 0.837 & 0.992 \\
$4 \times 8$ & 8.772 & 0.576 & 0.516 & 0.780 & 0.628 \\
$8 \times 16$ & 4.386 & 0.477 & 0.396 & 0.625 & 0.480 \\
$16 \times 32$ & 2.193 & 0.337 & 0.269 & 0.617 & 0.390 \\
$32 \times 64$ & 1.096 & 0.257 & 0.194 & 0.544 & 0.312 \\
$64 \times 128$ & 0.548 & 0.160 & 0.102 & 0.493 & 0.288 \\
\hline
\end{tabular}

Copyright $@$ ( ) by SIAM. Unauthorized reproduction of this article is prohibited. 

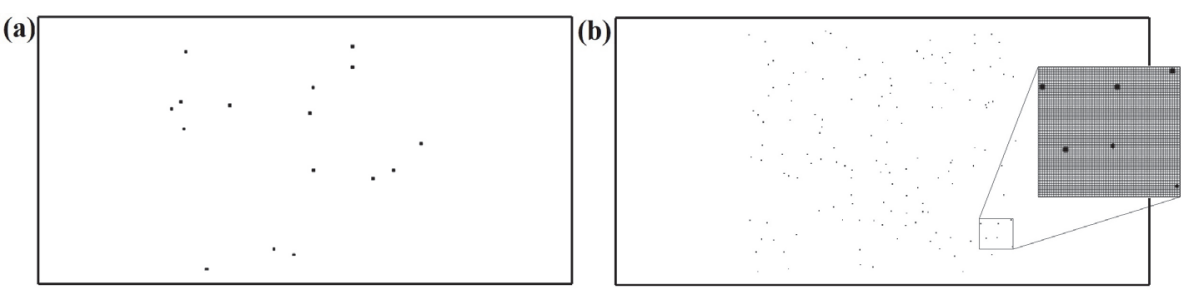

FIG. 6. The computational domains with arbitrarily placed obstacles for the open-channel flow tests. (a) Case A with 16 obstacles. (b) Case B with 144 obstacles (the enlarged figure is gridded to illustrate the size of fine obstacles with respect to fine elements).

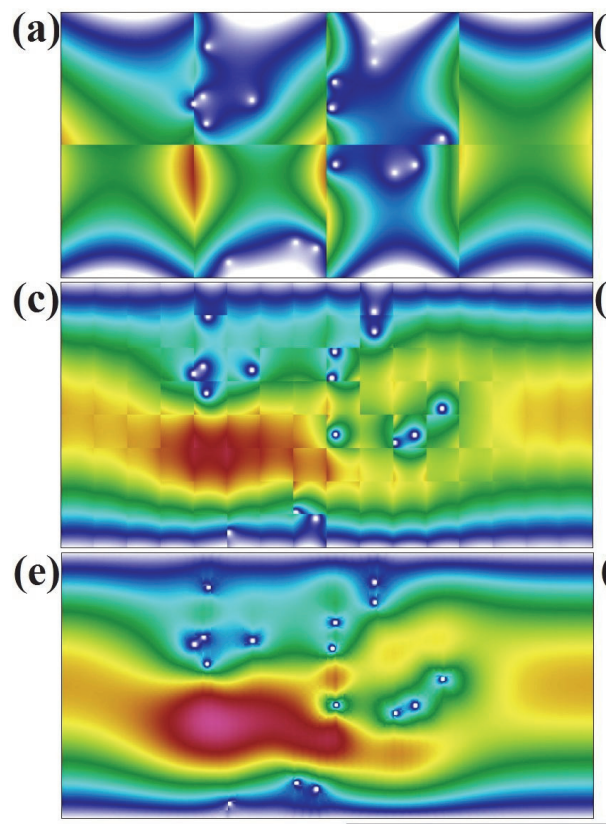

(g)

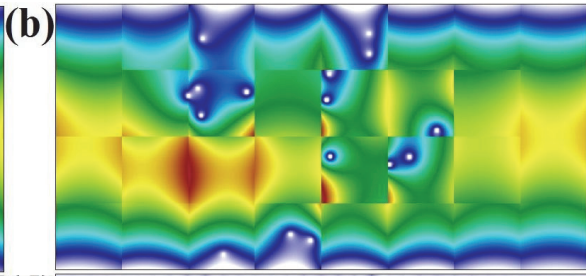

(d)

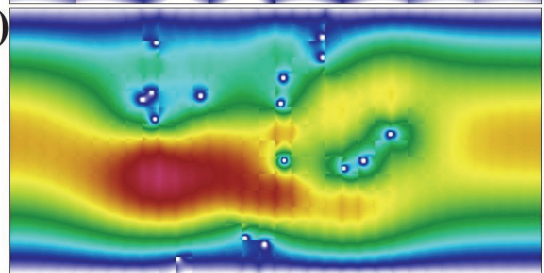

(f)

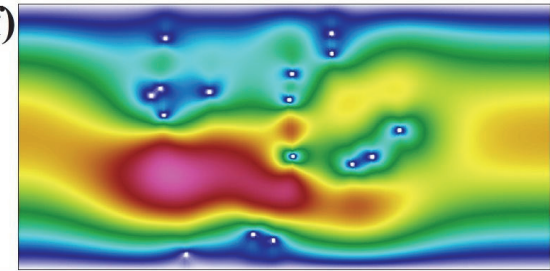

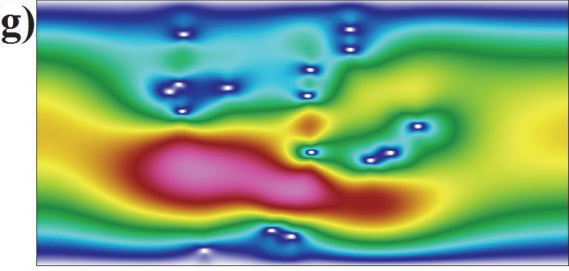

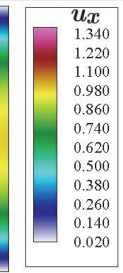

FIG. 7. The plots of the velocity $u_{x}$ contours of a channel flow computed on (a) $2 \times 4$, (b) $4 \times 8$, (c) $8 \times 16$, (d) $16 \times 32$, (e) $32 \times 64$, (f) $64 \times 128$ coarse elements. (g) The reference solution computed on $640 \times 1280$ elements. All have 16 arbitrarily placed obstacles.

Copyright (c) by SIAM. Unauthorized reproduction of this article is prohibited. 


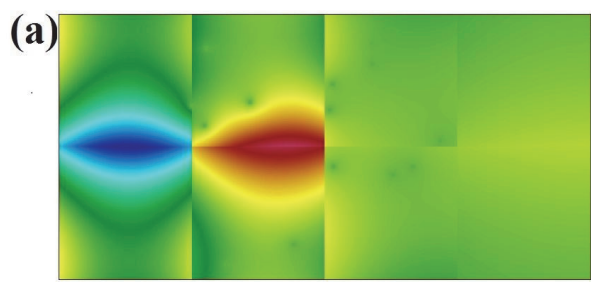

(c)
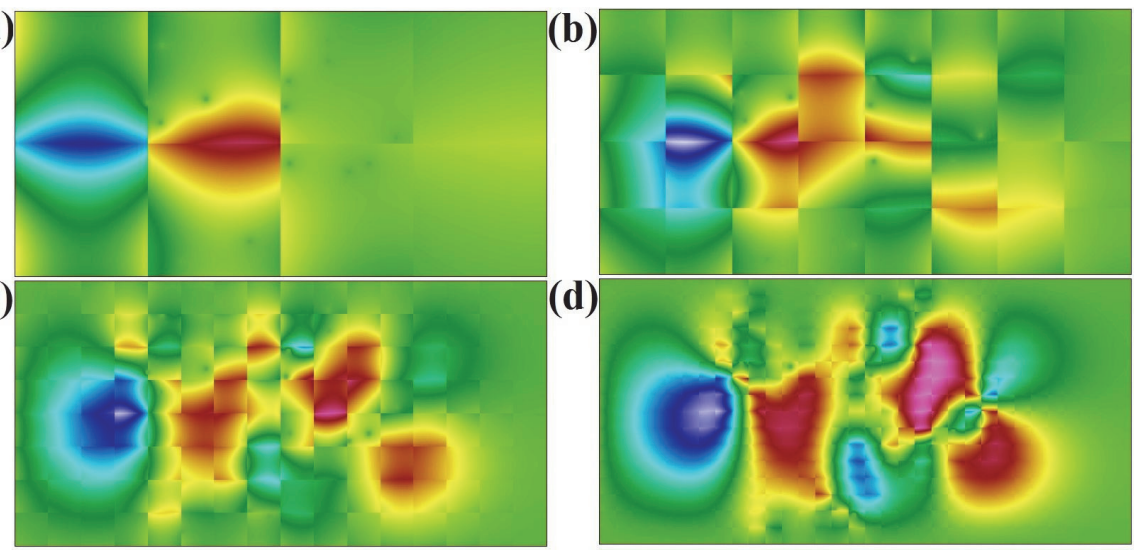

(e)
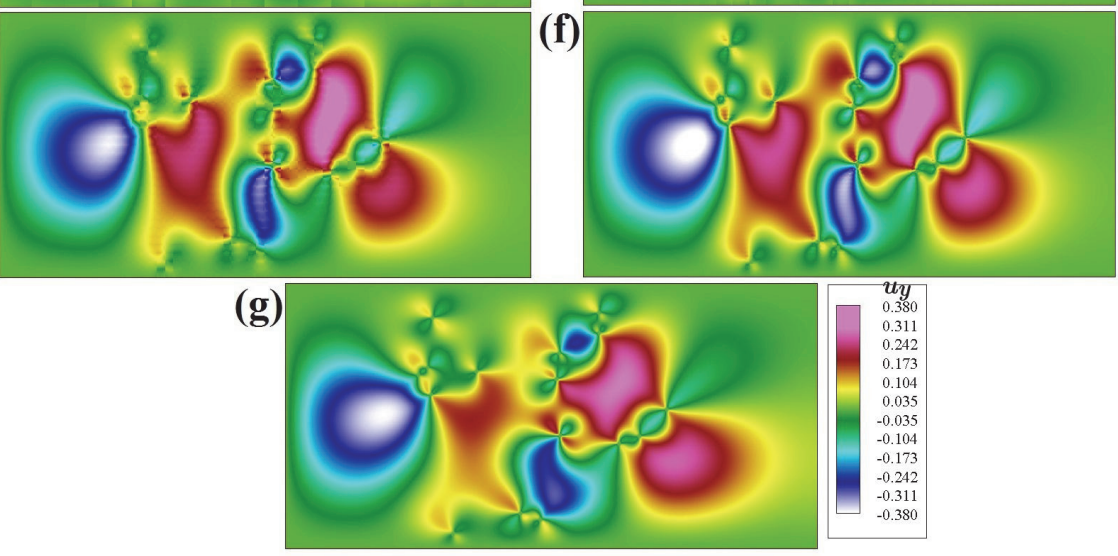

FIG. 8. The plots of velocity $u_{y}$ contours of a channel flow computed on (a) $2 \times 4$, (b) $4 \times 8$, (c) $8 \times 16$, (d) $16 \times 32$, (e) $32 \times 64$, (f) $64 \times 128$ coarse elements. (g) The reference solution computed on $640 \times 1280$ elements. All have 16 arbitrarily placed obstacles.

TABLE 2

The numerical study of convergence of a channel flow (Case A).

\begin{tabular}{lccccc}
\hline \hline Config. & $(H / \epsilon)$ & $L^{1}$ & $L^{2}$ & $H^{1}$ & $L^{2} \mathrm{P}$ \\
& Ratio & Relative error & Relative error & Relative error & \begin{tabular}{c} 
Relative error \\
\hline $2 \times 4$
\end{tabular} \\
25.00 & 0.305 & 0.395 & 0.631 & 0.874 \\
$4 \times 8$ & 12.50 & 0.169 & 0.212 & 0.605 & 0.601 \\
$8 \times 16$ & 6.250 & 0.110 & 0.142 & 0.594 & 0.563 \\
$16 \times 32$ & 3.125 & 0.090 & 0.115 & 0.506 & 0.420 \\
$32 \times 64$ & 1.563 & 0.067 & 0.087 & 0.411 & 0.275 \\
$64 \times 128$ & 0.781 & 0.043 & 0.062 & 0.320 & 0.141 \\
\hline
\end{tabular}

Copyright $@$ by SIAM. Unauthorized reproduction of this article is prohibited. 


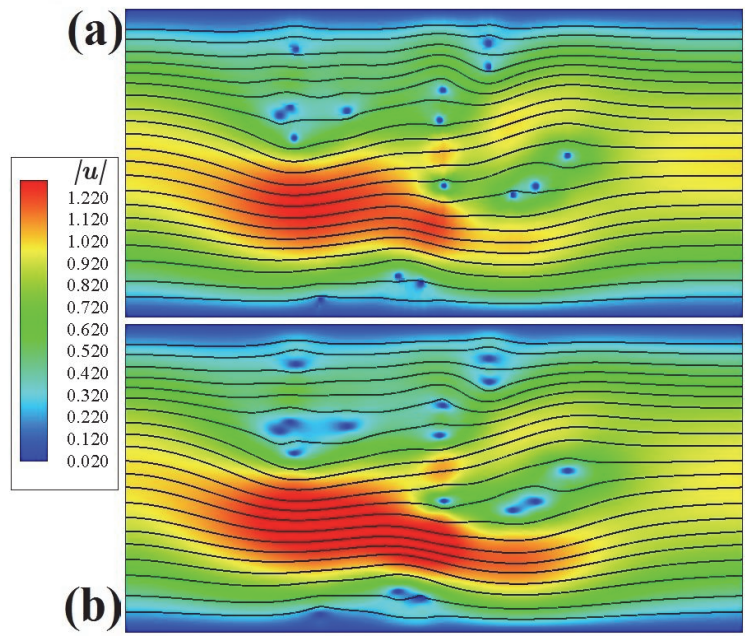

FIG. 9. The plots of velocity magnitude $|u|$ contours of a channel flow (Case A) complete with streamlines computed using Crouzeix-Raviart MsFEM on (a) $32 \times 64$ coarse elements, compared with (b) the reference solution computed on $1280 \times 640$ elements.
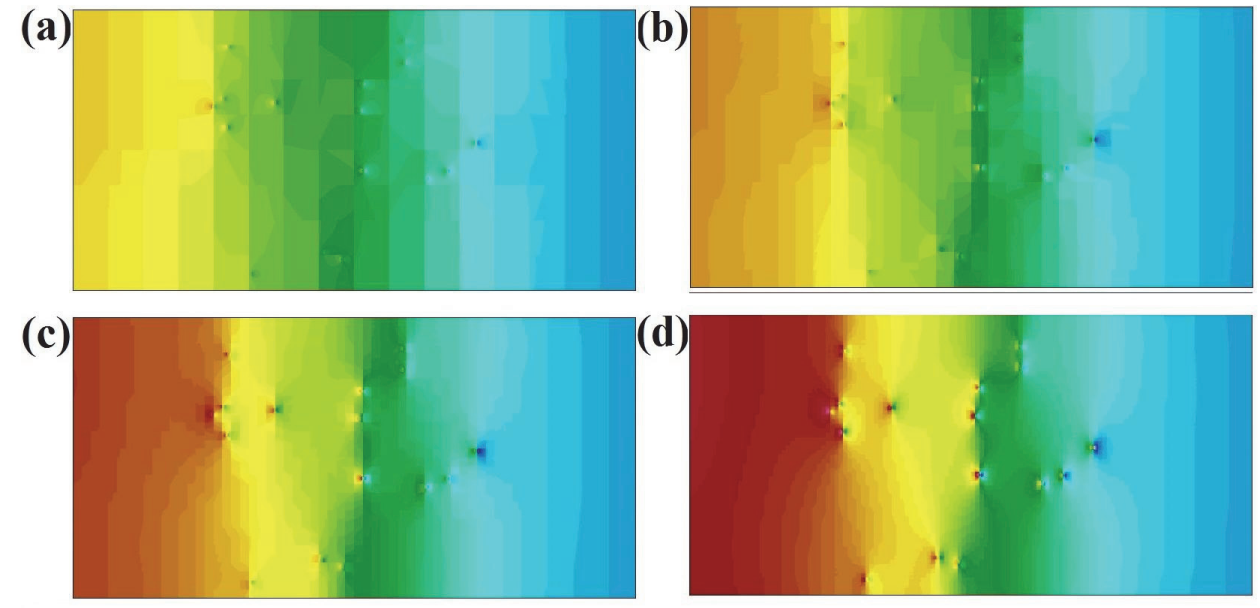

(e)
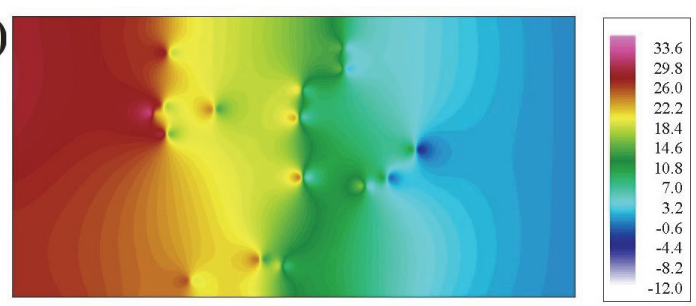

FIG. 10. The pressure contours for Case A computed on (a) $8 \times 16$, (b) $16 \times 32$, (c) $32 \times 64$, (d) $64 \times 128$ coarse elements, compared with (e) the reference solution computed on $1280 \times 640$ elements. 


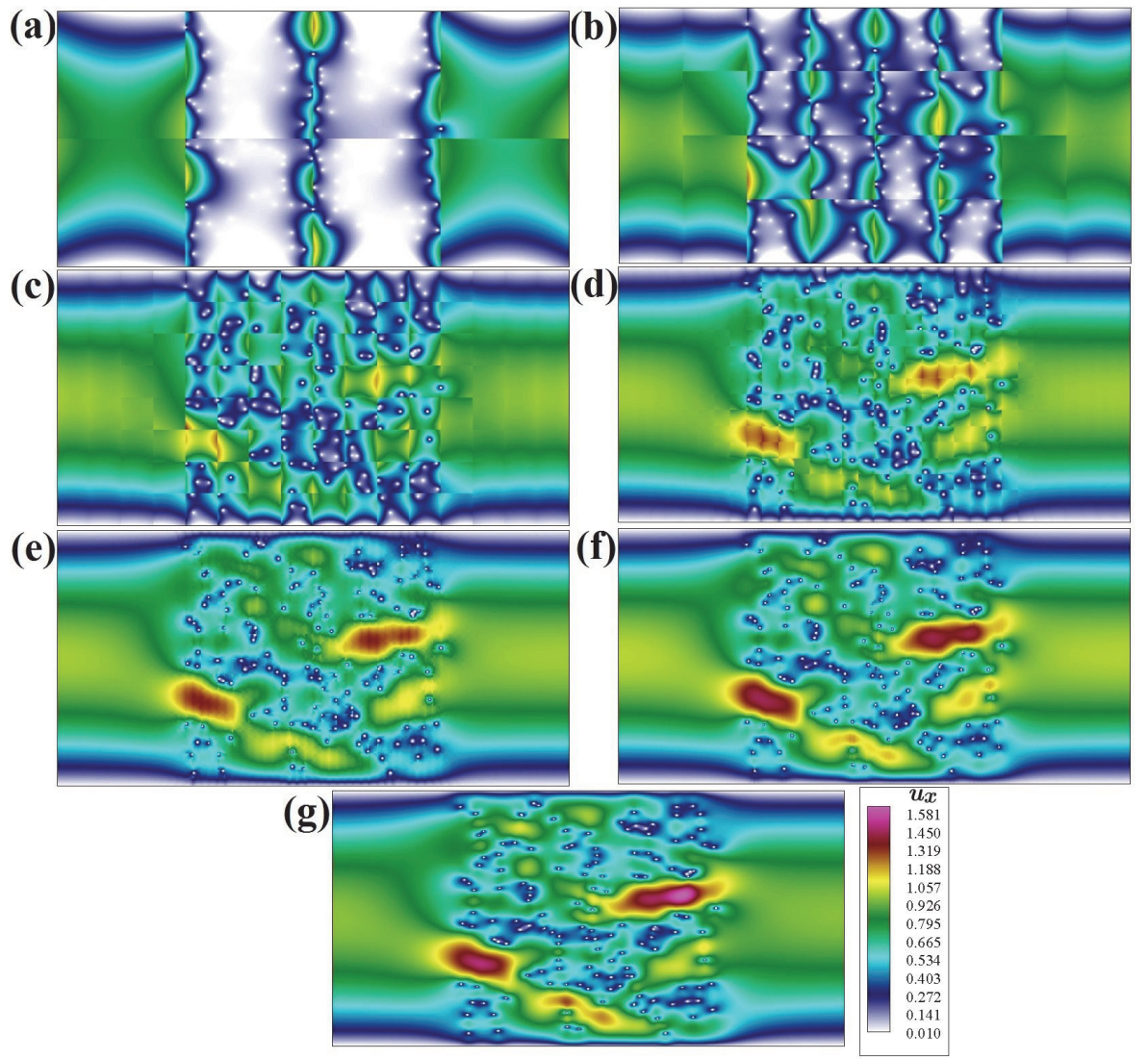

FIG. 11. The plots of velocity $u_{x}$ contours of a channel flow computed on (a) $2 \times 4$, (b) $4 \times 8$, (c) $8 \times 16$, (d) $16 \times 32$, (e) $32 \times 64$, (f) $64 \times 128$ coarse elements, and (g) the reference solution computed on $640 \times 1280$, with 144 arbitrarily placed obstacles. 

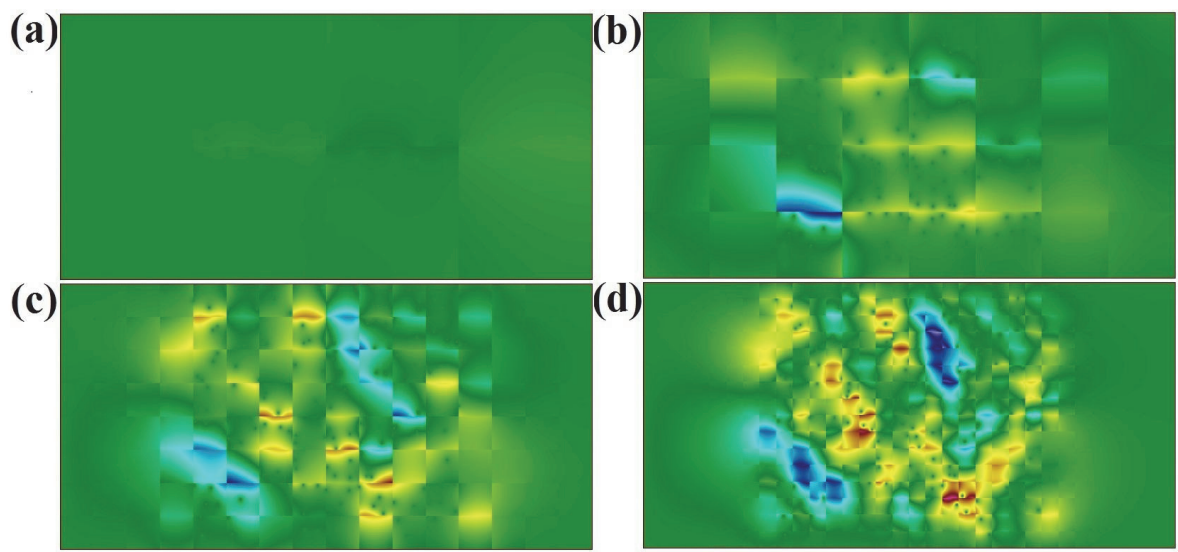

(e)

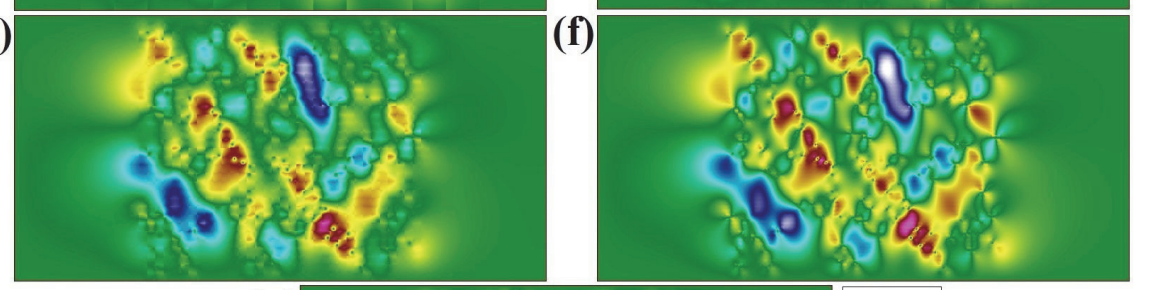

(g)

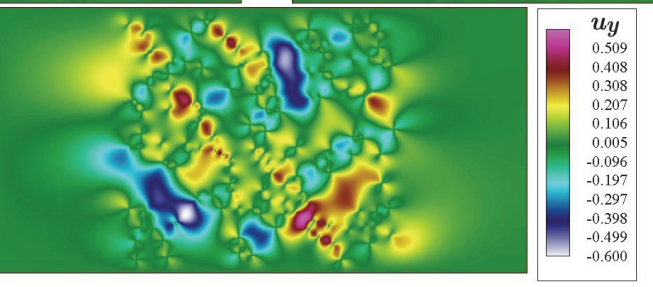

Fig. 12. The plots of velocity $u_{y}$ contours of a channel flow computed on (a) $2 \times 4$, (b) $4 \times 8$, (c) $8 \times 16$, (d) $16 \times 32$, (e) $32 \times 64$, (f) $64 \times 128$ coarse elements, and (g) the reference solution computed on $640 \times 1280$, with 144 arbitrarily placed obstacles. 


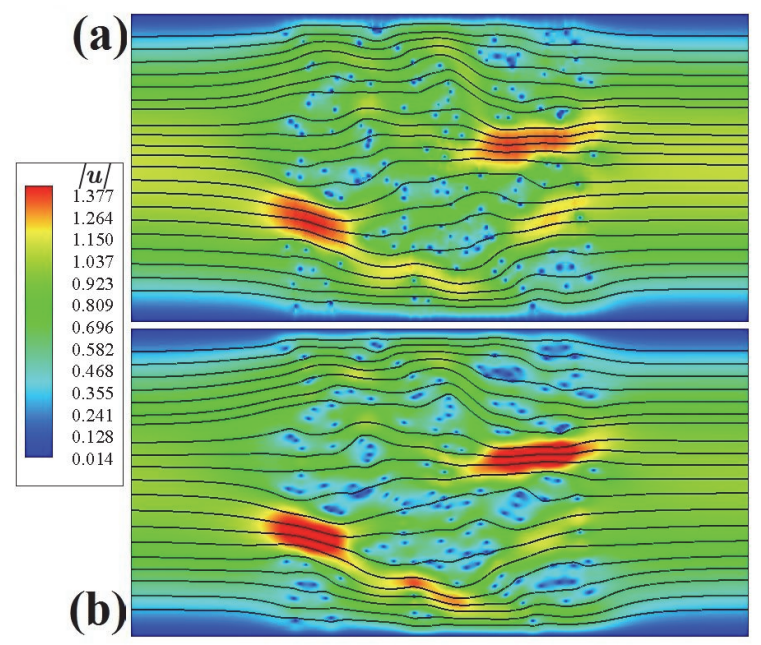

FIG. 13. The plots of velocity magnitude $|u|$ contours of a channel flow (Case B) complete with streamlines computed using Crouzeix-Raviart MsFEM on (a) $32 \times 64$ coarse elements, compared with (b) the reference solution computed on $1280 \times 640$ elements.

TABLE 3

The numerical study of convergence of a channel flow (Case B).

\begin{tabular}{|c|c|c|c|c|c|}
\hline Config. & $\begin{array}{l}(H / \epsilon) \\
\text { Ratio }\end{array}$ & $\begin{array}{c}L^{1} \\
\text { Relative error }\end{array}$ & $\begin{array}{c}L^{2} \\
\text { Relative error }\end{array}$ & $\begin{array}{c}H^{\mathrm{I}} \\
\text { Relative error }\end{array}$ & $\begin{array}{c}L^{2} \mathrm{P} \\
\text { Relative error }\end{array}$ \\
\hline $2 \times 4$ & 120.192 & 0.508 & 0.609 & 0.892 & 0.891 \\
\hline $4 \times 8$ & 60.096 & 0.321 & 0.423 & 0.805 & 0.800 \\
\hline $8 \times 16$ & 30.048 & 0.171 & 0.237 & 0.694 & 0.730 \\
\hline $16 \times 32$ & 15.024 & 0.104 & 0.144 & 0.606 & 0.666 \\
\hline $32 \times 64$ & 7.512 & 0.080 & 0.110 & 0.561 & 0.490 \\
\hline $64 \times 128$ & 3.756 & 0.062 & 0.081 & 0.452 & 0.259 \\
\hline
\end{tabular}

Copyright (c) by SIAM. Unauthorized reproduction of this article is prohibited. 


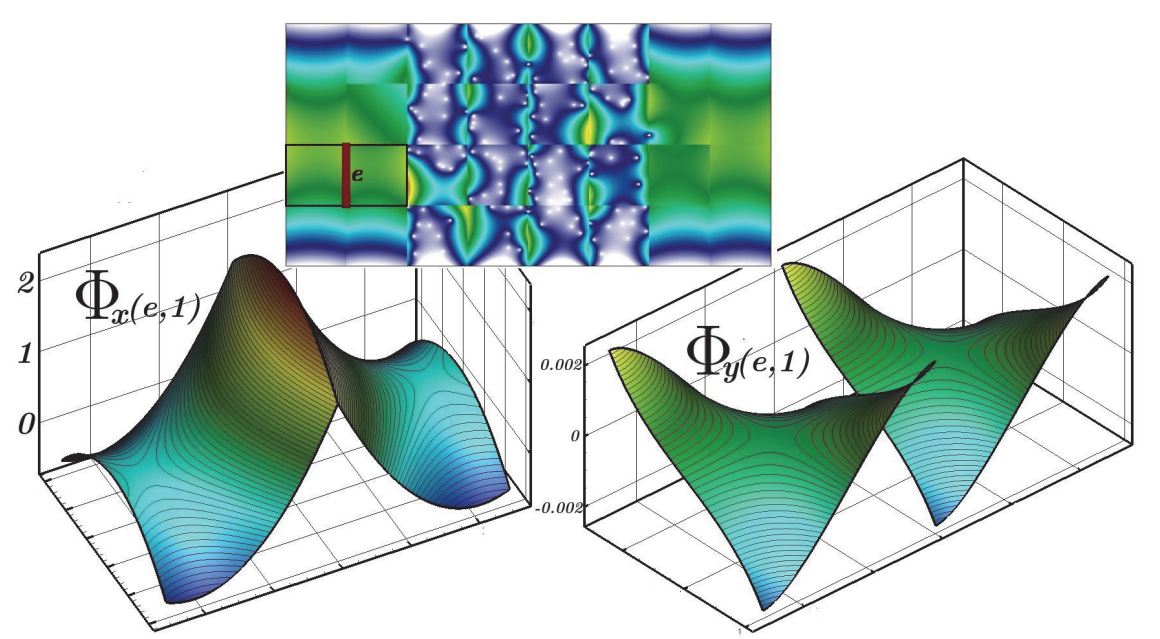

FIG. 14. Crouzeix-Raviart MsFEM basis function for elements without obstacles.

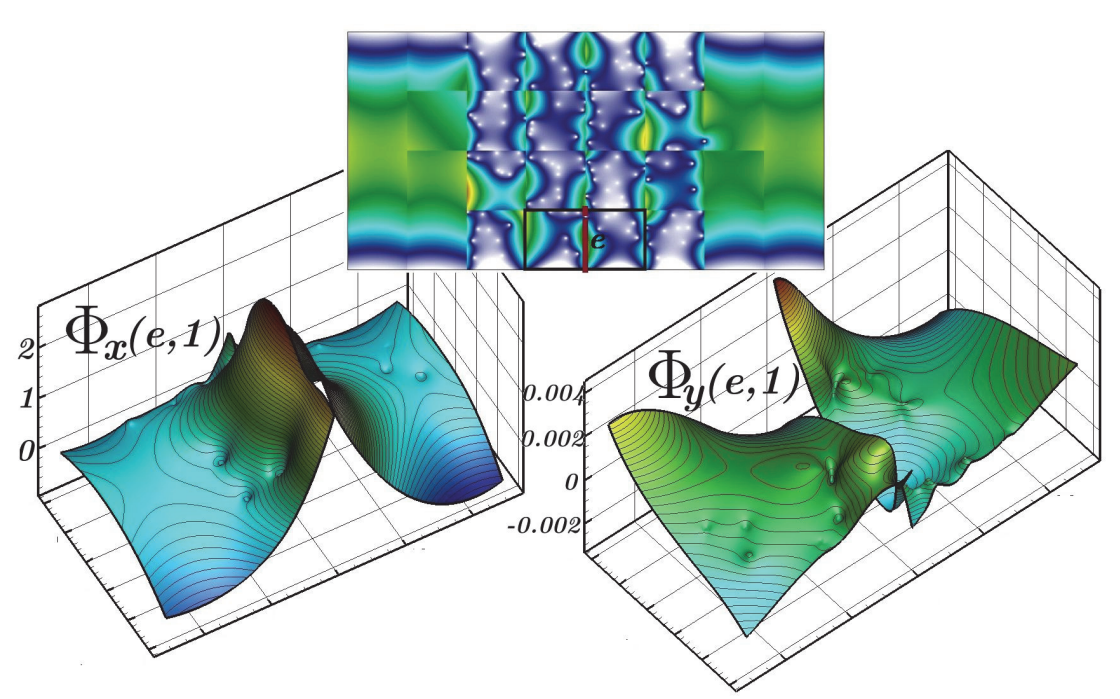

FIG. 15. Crouzeix-Raviart MsFEM basis function for elements with arbitrarily placed obstacles. 
6. Concluding remarks. The Crouzeix-Raviart MsFEM has been developed and tested for solving Stokes flow in genuine heterogeneous media. By "genuine" we mean in circumstances where analytical representation of the microscopic features of the flow is unavailable. This is illustrated by using very fine and nonperiodic placements of obstacles. The method has been tested in the context of obstacle-filled cavity flows and open-channel flows.

The Crouzeix-Raviart MsFEM basis functions are computed within each coarse elements using Q1-Q1 FEM. The nonconforming nature of the Crouzeix-Raviart element allows the method to accommodate a random pattern of obstacles without having to use oversampling methods.

Penalization method has been seamlessly incorporated in modeling arbitrary pattern of obstacles, thus allowing extensive use of a simple Cartesian mesh. Numerical studies of convergence of enclosed and open flows are given. Additionally, nonhomogeneous boundary conditions are considered in the test cases to demonstrate the robustness of the method. Good quantitative agreement has been reached with the reference solution at relatively coarse mesh configurations.

Although the test cases are given in two spatial dimensions, the extension of this work onto three spatial dimensions is straightforward. The calculations of MsFEM basis functions within a coarse element are done independent of its neighboring elements, which makes it suitable for the application of parallel programming.

\section{REFERENCES}

[1] T. Y. Hou AND X. H. Wu, A multiscale finite element method for elliptic problems in composite materials and porous media, J. Comput. Phys., 134 (1997), pp. 169-189.

[2] I. Babuška, U. Banerjee, And J. Osborn, Survey of meshless and generalized finite element methods: A unified approach, Acta Numer., (2003), pp. 1-125.

[3] M. Dorobantu and B. Engquist, Wavelet-based numerical homogenization, SIAM J. Numer. Anal., 35 (1998), pp. 540-559.

[4] J. Nolen, G. Papanicolaou, and O. Pironneau, A framework for adaptive multiscale methods for elliptic problems, Multiscale Model. Simul., 7 (2008), pp. 171-196.

[5] A. Bourgeat, Homogenized behaviour of two-phase flows in naturally fractured reservoirs with uniform fractures distribution, Comput. Methods Appl. Mech. Engrg., 47 (1984), pp. 205215.

[6] I. Kevrekidis, C. Gear, J. Hyman, P. Kevrekidis, O. Runborg, and C. Theodoropoulos, Equation-free, coarse-grained multiscale computation: Enabling microscopic simulators to perform system-level analysis, Commun. Math. Sci., 1 (2003), pp. 715-762.

[7] Weinan E and B. Engquist, The heterogeneous multi-scale methods, Comm. Math. Sci., 1 (2003), pp. 87-132.

[8] D. Cioranescu, P. Donato, And R. Zaki, Periodic unfolding and Robin problems in perforated domains, C. R. Acad. Sci. Paris, 342 (2006), pp. 469-474.

[9] D. Cioranescu and F. Murat, A strange term coming from nowhere, in Topics in the Mathematical Modelling of Composite Materials, Progr. Nonlinear Differential Equations Appl. 31, Birkhäuser, Basel, 1997, pp. 45-93.

[10] P. Henning And M. OhlBerger, The heterogeneous multiscale finite element method for elliptic homogenization problems in perforated domains, Numer. Math., 113 (2009), pp. 601629.

[11] U. Hornung, Homogenization and Porous Media, Interdiscip. Appl. Math. 6, Springer-Verlag, New York, 1997.

[12] J.-L. Lions, Asymptotic expansions in perforated media with a periodic structure, Rocky Mountain J. Math., 10 (1980), pp. 125-140.

[13] W. Deng, X. Yun, And C. XIE, Convergence analysis of the multiscale method for a class of convection-diffusion equations with highly oscillating coefficients, Appl. Numer. Math., 59 (2009), pp. 1549-1567.

[14] P. Henning and M. Ohlberger, The heterogeneous multiscale finite element method for advection-diffusion problems with rapidly oscillating coefficients and large expected drift, Netw. Heterog. Media, 5 (2010), pp. 711-744. 
[15] A. Abdulle and M. Huber, Discontinuous Galerkin finite element heterogeneous multiscale method for advection-diffusion problems with multiple scales, Numer. Math., 126 (2014), pp. 589-633.

[16] D. Brown, Y. Efendiev, G. Li, P. Popov, and V. Savatorova, Multiscale modeling of high contrast Brinkman equations with applications to deformable porous media, in Poromechanics V: Proceedings of the Fifth Biot Conference on Poromechanics, Vienna, Austria, 2013, pp. 1991-1996.

[17] D. L. Brown, Y. Efendiev, and V. H. HoAng, An efficient hierarchical multiscale finite element method for Stokes equations in slowly varying media, Multiscale Model. Simul., 11 (2013), pp. 30-58.

[18] T. Arbogast and H. Lehr, Homogenization of a Darcy-Stokes system modeling vuggy porous media, Comput. Geosci., 10 (2006), pp. 291-302.

[19] V. Girault, D. Vassilev, and I. Yotov, Mortar multiscale finite element methods for StokesDarcy flows, Numer. Math., (2013), pp. 1-73.

[20] A. F. Gulbransen, V. L. Hauge, and K. A. Lie, A multiscale mixed finite-element method for vuggy and naturally fractured reservoirs, Soc. Petroleum Engineers J., 15 (2010), pp. 395403.

[21] P. Popov, Y. Efendiev, AND G. QIn, Multiscale modeling and simulations of flows in naturally fractured Karst reservoirs, Commun. Comput. Phys., 6 (2009), pp. 162-184.

[22] Y. Efendiev And T. Y. Hou, Multiscale Finite Element Method. Theory and Applications, Surv. Tutor. Appl. Math. Sci. 4, Springer, New York, 2009.

[23] J. Chu, Y. Efendiev, V. Ginting, and T. Hou, Flow based oversampling technique for multiscale finite element methods, Adv. Water Res., 31 (2008), pp. 599-608.

[24] P. Henning and D. Peterseim, Oversampling for the multiscale finite element method, Multiscale Model. Simul., 11 (2013), pp. 1149-1175.

[25] Y. Efendiev, J. Galvis, G. Li, And M. Presho, Generalized multiscale finite element methods. Oversampling strategies, Internat. J. Multiscale Comput. Engrg., 12 (2014), pp. 465-484.

[26] M. CRouzeix And P. A. Raviart, Conforming and nonconforming finite element methods for solving the stationary Stokes equations I, RAIRO, 7 (1973), pp. 33-75.

[27] C. Le Bris, F. Legoll, And A. Lozinski, MsFEM à la Crouzeix-Raviart for highly oscillatory elliptic problems, Chin. Ann. Math. Ser. B, 34 (2013), pp. 113-138.

[28] C. Le Bris, F. Legoll, And A. Lozinski, An msFEM type approach for perforated domains, Multiscale Model. Simul., 12 (2014), pp. 1046-1077.

[29] P. Degond, A. Lozinski, B. P. Muljadi, And J. NArski, Crouzeix-Raviart MsFEM with bubble functions for diffusion and advection-diffusion in perforated media, Commun. Comput. Phys., 17 (2015), pp. 887-907.

[30] V. Girault And P.-A. Raviart, Finite Element Methods for Navier-Stokes Equations. Theory and Algorithms, Springer Ser. Comput. Math. 5, Springer-Verlag, Berlin, 1986.

[31] A. Ern and J.-L. Guermond, Theory and Practice of Finite Elements, Appl. Math. Sci. 159, Springer-Verlag, New York, 2004.

[32] G. JANKOWIAK AND A. LozINSKI, Non-conforming multiscale finite element method for Stokes flows in heterogeneous media. Part II: Error estimates for periodic microstructure, in preparation.

[33] P. Angot, C.-H. Bruneau, And P. Fabrie, A penalization method to take into account obstacles in incompressible viscous flows, Numer. Math., 81 (1999), pp. 497-520.

[34] L. Carballal Perdiz, Etude d'une méthodologie multiéchelles appliquée à différents problèmes en milieu continu et discret, Ph.D. thesis, Institut de Mathématiques de Toulouse, 2011.

[35] F. Brezzi And M. Fortin, Mixed and Hybrid Finite Element Methods, Springer Ser. Comput. Math. 15, Springer-Verlag, New York, 1991.

[36] F. Brezzi and J. Pitkaranta, On the stabilization of finite element approximations of the Stokes problem, in Efficient Solutions of Elliptic Systems, Notes Numer. Fluid Mech. 10, 1984, Viewig+Teubner Verlag, Berlin, pp. 11-19.

[37] H. C. Elman, D. J. Silvester, And A. J. Wathen, Finite Elements and Fast Iterative Solvers: With Applications in Incompressible Fluid Dynamics, Numer. Math. Sci. Comput., Oxford University Press, Oxford, UK, 2005. 